\title{
ON THE RELATIONSHIP BETWEEN PLANE AND SOLID GEOMETRY
}

\author{
ANDREW ARANA AND PAOLO MANCOSU \\ Department of Philosophy of Kansas State University and \\ University of California-Berkeley
}

\begin{abstract}
Traditional geometry concerns itself with planimetric and stereometric considerations, which are at the root of the division between plane and solid geometry. To raise the issue of the relation between these two areas brings with it a host of different problems that pertain to mathematical practice, epistemology, semantics, ontology, methodology, and logic. In addition, issues of psychology and pedagogy are also important here. To our knowledge there is no single contribution that studies in detail even one of the aforementioned areas.

In this paper our major concern is with methodological issues of purity and thus we treat the connection to other areas of the planimetry/stereometry relation only to the extent necessary to articulate the problem area we are after.

Our strategy will be as follows. In the first part of the paper we will give a rough sketch of some key episodes in mathematical practice that relate to the interaction between plane and solid geometry. The sketch is given in broad strokes and only with the intent of acquainting the reader with some of the mathematical context against which the problem emerges. In the second part, we will look at a debate (on "fusionism") in which for the first time methodological and foundational issues related to aspects of the mathematical practice covered in the first part of the paper came to the fore. We conclude this part of the paper by remarking that only through a foundational and philosophical effort could the issues raised by the debate on "fusionism" be made precise. The third part of the paper focuses on a specific case study which has been the subject of such an effort, namely the foundational analysis of the plane version of Desargues' theorem on homological triangles and its implications for the relationship between plane and solid geometry. Finally, building on the foundational case study analyzed in the third section, we begin in the fourth section the analytic work necessary for exploring various important claims about "purity," "content," and other relevant notions.
\end{abstract}

\section{§1. Mathematical practice.}

1.1. The Greeks. If we look at Euclid's Elements we find a separation between plane (Books I-IV, and arguably VI and X) and solid geometry (books XI-XIII). The separation is not that neat, for in the stereometric books Euclid establishes many results that pertain to plane geometry: for instance 12 out of the 18 theorems of book XIII are theorems of plane geometry. While elements of plane geometry are obviously needed in the development of solid geometry, Euclid has no applications of solid geometry in establishing results of plane geometrical statements. Nevertheless, the application of stereometric considerations for solving problems of plane geometry was not unknown to the Greeks. Such constructions or demonstrations using solid geometry to establish results of plane geometry remained confined to the advanced branches of the subject, however, as opposed to the elementary

Received: January 10, 2011. 
treatments (such as Euclid's). There are five areas of geometry in which, up to the time of Euclid, stereometrical considerations appear: ${ }^{1}$

i) Astronomical studies related to the hypotheses on the sphericity of the universe, of the earth and the planets, and on the circularity of the celestial movements; ${ }^{2}$

ii) The study of regular polyhedra (also in connection to astronomy) and their "isoepiphan" properties (analogues to the isoperimetric properties for regular polygons);

iii) The solution to the problem of the duplication of the cube and its reduction-generalization to that of the insertion of two mean proportionals between two line segments;

iv) The study of conic sections (or sections of the cone);

v) Problems of cubature in the sense of measurements of volumes.

Beyond Euclid we have, in addition to developments in the above five areas, applications of stereometric considerations in the form of projections of curves defined on surfaces (such as a cylinder or a sphere) in the study of properties of curves such as the spiral and the quadratrix (cf. Mancosu \& Arana, 2010, §4.2; Knorr, 1993, pp. 166-167).

Of interest to us, in the list above, are areas iii and iv. While the duplication of the cube is obviously a stereometric problem, the determination of two mean proportionals between two line segments is not. However, solving this problem led several mathematicians to exploit the resources of stereometry, at least implicitly. It is worthwhile to recall two wellknown solutions. The first is that of Archytas (fourth century B.C.) who, according to Eutocius' commentary on the sphere and the cylinder (see Archimedes, 2004, pp. 290293), solves the problem of finding two mean proportionals to two given line segments by a construction that requires appeal to stereometric considerations. ${ }^{3}$ The second solution is due to Maenechmus and requires the intersection of two conic sections (translation in Archimedes, 2004, pp. 286-290). ${ }^{4}$ Since conic sections are obtained through sections of the cone the solution of the problem seems to rest, at least implicitly, on stereometric considerations. $^{5}$

In order to avoid misunderstandings, it is important to remark here that we do not intend to claim that the distinction between planimetric and stereometric considerations was at the center of Greek foundational thinking. Much more important for the Greek mathematical

1 Here we follow Vitrac (2000), pp. 19-24.

2 Related to this is the issue of the relationship between spherical and plane trigonometry. For instance astrolabes were instruments used to represent on the plane by means of projections the movements of celestial phenomena occurring in three-dimensional space.

3 For an exposition of the result the reader is referred to Heath (1921), pp. 246-249. For Archytas' proof see also the analysis by F. Acerbi in Euclid (2007), pp. 79-87.

4 For an exposition of the result the reader is referred to Heath (1921), pp. 251-255. For Maenechmus' proof see also the analysis by F. Acerbi in Euclid (2007), pp. 99-104.

5 Of course, a construction of the conic sections that does not generate them as sections of the cone but rather directly on the plane would not be dependent on stereometry. For instance, Diocles' construction of the parabola in On Burning Mirrors, propositions 4 and 5, is given by a pointwise construction that does not require stereometric considerations. In this connection it is relevant that $\mathrm{Ph}$. de La Hire in his 1673 treatise Nouvelle méthode en Géométrie, pour les sections de superficies coniques et cylindrique. Planiconiques, and more specifically in the second part Planiconiques, developed the theory of conic sections in the plane without going through sections of the cone. As the reviewer of the Journal des Savants in 1674 pointed out, 
tradition was the distinction between problems, as reported by Pappus, into plane, solid, and linear. The distinction classifies problems according to the types of curves necessary for their solution. Plane problems require only straight lines and circles (ruler and compass constructions); solid problems require an appeal to conic sections; linear problems require the use of curves of more complicated genesis (quadratrix, conchoids etc.). While one might be tempted to narrowly connect the notion of solid problem to the use of stereometry, it would be unwise to do so. Indeed, according to Pappus' partition, all the stereometry presented by Euclid in books XI-XIII is "plane." Moreover, "solid problems" are not seen by a Greek mathematician as essentially pertaining to stereometry; rather, as we will soon indicate, planimetric problems can be "solid."

Having said that, it remains true that at least implicitly (as in the use of conic sections) or explicitly (as in the case of Archytas) some of the solutions to the problem of finding two mean proportionals made appeal to stereometric objects.

While Euclid has no uses of stereometry for the solution of planimetric problems, we are also not aware of any explicit methodological qualms in the Greek tradition against such a possibility. ${ }^{6}$ Indeed, if the appeal to conic sections can be construed as implicitly relying on stereometry (on account of the use of the cone to generate the conic sections) then it is easy to give examples of such. For instance, within the classification of problems provided by Pappus, a problem like the trisection of an angle (whose statement is planimetric) is "solid," for it requires the appeal to conic sections, which require a stereometric object (a cone) for their generation. In other words, problems whose statement involves only notions of plane geometry need not be "plane" problems according to Pappus' classification.

A similar situation is encountered when Pappus also describes constructions by projections. ${ }^{7}$ For instance, Pappus gives the quadrature of the circle, a problem whose statement is planimetric, using the quadratrix. However, in the attempt to provide a "less mechanical" construction of the quadratrix, Pappus (1876-1878, vol. 1, pp. 262-265) obtains the latter by projection of a cylindrical helix - a curve constructed on a cylinder. Once the quadratrix is generated in this way, one can then go on to square the circle using the properties of the quadratrix. In this case the problem is "linear" and depends obviously on stereometric considerations.

Thus, we have seen that during the Greek period the use of stereometric means for the solution of problems of plane geometry was limited to the more advanced areas of the subject and that no explicit criticism seems to have been explicitly raised against such

Mais parce que tous les Geometres ne sont pas accoûtumez à concevoir des solides par des simples lignes tracées sur un plan, cet Auteur a ajoûté à sa nouvelle Methode un traité des Planiconiques; qui est tres-beau \& tres-commode, puisque par là il n'est plus besoin d'imaginer aucun solide ny plan que celuy sur lequel est la figure. (p. 11)

While there is no explicit commitment to an injunction of "purity," it is nonetheless the case that the elimination of considerations of solids or planes in which the conic section does not lie is seen by the reviewer as a positive achievement.

${ }^{6}$ Kreisel's reference to "the time of the Greeks when Archimedes was criticized for using properties of space to prove theorems about the plane" (cf. Kreisel, 1980, p. 163) is a mistake, wrongly taking Pappus' criticisms of "solid" solutions of plane problems to be criticisms of stereometric solutions of plane problems. Kreisel thus mixed up Pappus' technical use of the term "solid" with its more ordinary use referring to space.

7 Later examples would include the study by John Collins and Jacob Bernoulli of the logarithmic spiral, an object in two dimensions, as a projection of the loxodrome, a curve in space. 
uses. However, the structure of Euclid's Elements, which limits solid geometry to books XI-XIII, and the fact that later on those books were less studied than the planimetric books, had an important effect in keeping stereometrical considerations separate from planimetric investigations. As we shall see later, even at the time of Gergonne one bemoaned the separation of plane and solid geometry as counterproductive.

1.2. Some examples from the seventeenth century. It is in the first half of the seventeenth century that a deeper interaction between plane and solid geometry seems to have emerged in mathematical practice. For instance, Luca Valerio, in Quadratura parabolae per simplex falsum (cf. Valerio, 1606), gave a new proof of the quadrature of the parabola, a classical result proved by Archimedes. He did so by exploiting a previous lemma on the center of gravity of plane figures composed by two semiparabolas. The proof of this lemma appealed to results from solid geometry by extracting information about the center of gravity of the plane figure through considerations related to the center of gravity of an hemisphere. While it would be interesting to analyze in detail this result and its place in the context of Valerio's work, we restrict our focus instead to the work of a more representative mathematician, Evangelista Torricelli.

Torricelli knew Valerio's work quite well, and his case is especially interesting because he applies solid geometry in the solution of plane problems both in the context of classical mathematical techniques (namely, those inherited from Greek geometry) as well as in his creative applications of indivisibilist techniques (which originated with Cavalieri). Of great importance in this connection is the treatise De quadratura parabolae contained in the Opera Geometrica (cf. Torricelli, 1919-1944) and published in 1644. The treatise is a tour de force presenting 20 different proofs of the quadrature of the parabola. Recall that the quadrature of the parabola is the problem of determining the area of the parabolic segment obtained by cutting a parabola with a line, namely determining the space enclosed by the parabola and the chord cut off by the line. The theorem says that the parabolic segment is to the area of a certain triangle inscribed in the parabolic segment as 4 is to 3 .

Torricelli divides the treatment into two different parts. The first part presents various quadratures obtainable by classical techniques (double reductio ad absurdum). Of these, the quadratures given in propositions 8, 9, and 10 make use of solid techniques, as they involve the consideration of properties of cones, cylinders, and hemispheres. The strategy involves transforming a plane configuration (consisting of the segment of the parabola with additional geometrical plane objects such as parallelograms, triangles and other figures) by seeing it as the section of a solid configuration or by rotating it as to obtain a solid figure (involving cones, spheres, cylinders, and other more complex solids) and then inferring (either directly or by reductio ad absurdum with a previously known theorem of solid geometry) that the parabolic segment must be to the inscribed triangle as 4 is to 3 .

But it is in the second part of the treatise, where Torricelli displays the fruitfulness of the technique of indivisibles, that the application of solid techniques is truly pervasive. In fact, introducing the section on quadratures of the parabola given by indivisibilist techniques, Torricelli emphasizes his interest in inferring the quadrature of the parabola from certain classical Euclidean and Archimedean results in solid geometry:

So far we have treated the measurement of the parabola with the method of the ancients. We must now attack the same problem with a new and admirable method, namely the geometry of indivisibles, and in diverse ways. Indeed, if we take for granted the major theorems of the ancients, those of Euclid as well as those of Archimedes, even though they are 
about very different subject matters, it is surprising that the quadrature of the parabola can be deduced from each one of them with a simple procedure... Granted for instance that the cylinder is three times the cone inscribed in it, from this it follows that the parabola is to the inscribed triangle as 4 is to 3 . If instead one prefers to start from the theorem that says that the cylinder is to the inscribed sphere as 3 to 2 then one can immediately infer the quadrature of the parabola. (Torricelli, 1919-1944, p. 381 of Italian translation).

Consider, for instance, the quadrature given in proposition 11, where we consideraccording to the main intuition of the theory of indivisibles - cones and cylinders as being constituted by their plane sections. We begin with a parabola $A B C$, a tangent $C D$ and a parallel $A D$ to the diameter of the parabola (as in Figure 1).

We then consider the parallelogram $A D E C$ and construct a cone with base a circle with diameter $D A$ and vertex in $C$. At the same time we also consider a cylinder with one base identical with that of the cone (namely, the circle with diameter $D A$ ) and the other base the circle with diameter $E C$. Consider now an arbitrary segment $F G$ parallel to $D A$ (or $E C$ ) and the corresponding circle of which $F G$ is a diameter. Through some standard geometrical results (including properties of the parabola), Torricelli shows that for an arbitrary $F G$ (and the points $I, B$ it generates on the tangent and the parabola) we have that $F G$ is to $I B$ as the circle with diameter $F G$ is to the circle with diameter $I G$. The collection of all $F G$ s is nothing other than the parallelogram $A D E C$ while the collection of all circles with diameter $F G$ yields the cylinder (as $F G$ is an arbitrary section of $A D E C$ ). The collection of all the circles with diameter $I G$ is the cone with same base and height as the cylinder $A E$. But by Euclid XII.10 the ratio of a cone and a cylinder with same base and height is 1 to 3 . That means that the ratio of the parallelogram $A D E C$ (the collection of all the $F G \mathrm{~s}$ ) is to the figure $A B C D$ (the collection of all $I B \mathrm{~s}$ ) as 3 is to 1 . From there one can infer, but we omit to do so, the result that the area of the parabolic segment is to the inscribed triangle (not shown in Figure 1) as 4 is to 3.

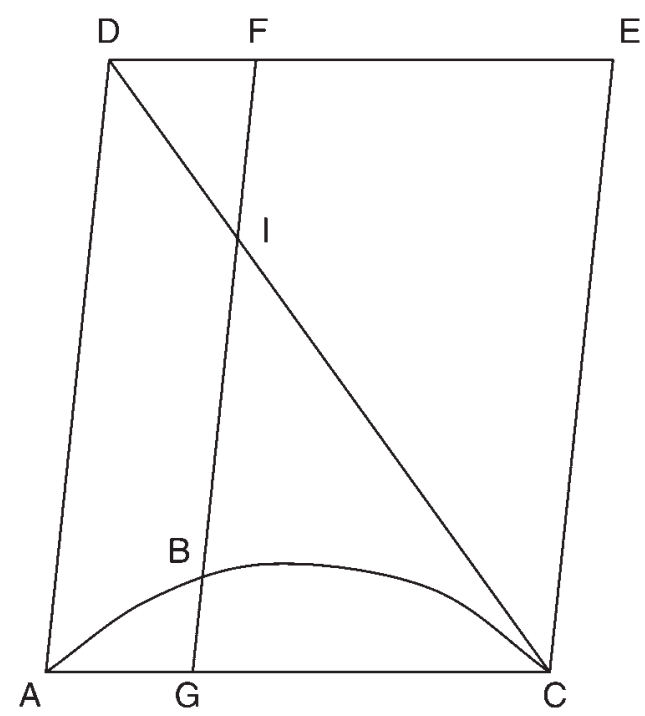

Fig. 1. Torricelli's quadrature of the parabola in proposition 11. 


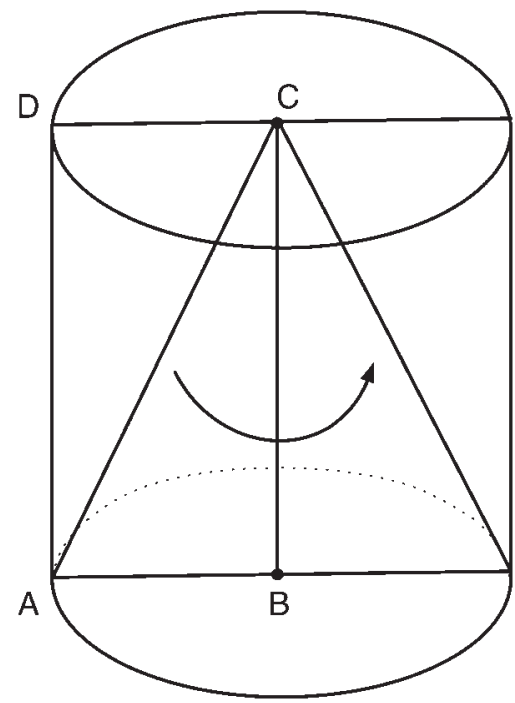

Fig. 2. Generation by rotation of the cone and cylinder from the rectangle and the triangle.

The exploitation of solid geometry in the determination of area problems (which must be seen as planimetric) is also found in Wallis' Arithmetica Infinitorum (1655), also a major text in the indivisibilist tradition (see Panza \& Malet, 2010). Perhaps the deep connection between plane and solid problems in the indivisibilist context might not be completely surprising as Cavalieri's geometry of indivisibles can be seen as constitutively tied to solid considerations. ${ }^{8}$ Indeed, Cavalieri himself explains in the preface to the Geometria (cf. Cavalieri, 1635) that he had been led to the geometry of indivisibles by the puzzling fact that the volumes of solid figures were not in the same proportion as that of the generating plane figures. For instance, Cavalieri noticed that if we consider a rectangle and one of the triangles obtained by joining two opposite vertices of the rectangle (as in Figure 2), the respective areas are as 2 to 1 .

By rotating the figures around the height of the rectangle we obtain a cylinder (generated by the rectangle $A B C D$ ) and a cone (generated by the triangle $\triangle A B C$ ) whose volumes are as 3 to 1 . Cavalieri was then able to reestablish a certain analogy between the plane case and the solid case by considering all the "planes" of the cone and all the "planes" of the cylinder. ${ }^{9}$

1.3. The school of Monge. The key example we will discuss at length later is the proof of Desargues' theorem on homological triangles in two dimensions making use of the same theorem in three dimensions. This proof is one of the standard examples of use of solid techniques in proofs of plane theorems. However, this is not the way Desargues proved the two-dimensional version of his theorem.

It is well known that Desargues' works were not easy to find, even in the seventeenth century. For instance, the Brouillon project d'une atteinte aux evenements des rencontres $d u$ cone avec un plan (1639) was published in only 50 copies and had already become

8 Newton had also used such proofs; cf. Whiteside (1967, I, 3, §2, pp. 91-95).

9 See also Palmieri (2009) for diagrams reconstructing Cavalieri's appeal to "all the squares" with three-dimensional diagrams. 
unobtainable by the time Leibniz looked for it in Paris in 1675 (see Taton, 1951a, 1951c). Until the rediscovery of a copy in 1951, one had relied on some passages in Bosse's Manière Universelle de M. Desargues pour pratiquer la perspective (1648) and on the rediscovery in 1845 by Chasles of Ph. de La Hire's handwritten copy of one part of the Brouillon. In particular, Desargues' theorem on homological triangles in projective geometry, which we discuss in detail later, might have been contained in a lost treatise by Desargues with the title Leçons de ténèbres (see Gray \& Field, 1987, p. 161). It became known through Bosse's treatise La perspective de Mr Desargues (1648, pp. 340-341; English translation in Gray \& Field, 1987, pp. 160-164). Bosse reports there, in Proposition I, two proofs given by Desargues, one for two homological triangles lying in different planes in space and one, based on Menelaus' theorem, for the two-dimensional case. Thus, Desargues here does not use the solid version of his result when proving the plane version. This deserves to be pointed out, for a superficial glance at some classic sources in the literature might be misleading. For instance, Chasles in his classic Aperçu (1837) cites a passage from Bosse's Traité de pratiques géométrales et perspectives (1665):

M. Desargues proved universally by means of solids, and this is not the ordinary practice of all those who claim to be geometers or mathematicians (cited by Chasles, 1837, p. 87)

As we have seen, Desargues' theorem on homological triangles, at least as reported by Bosse, was not an instance of such proofs "by means of solids" and indeed we do not seem to have any such proofs in the Desarguian corpus. ${ }^{10}$ Nonetheless, Chasles used the above quote to conjecture that Desargues might have anticipated the characteristic feature of Monge's work in projective geometry and his school at the beginning of the nineteenth century. Indeed, commenting on Bosse's passage he went on to say:

Couldn't these words by Bosse, by means of solids, signify that Desargues used in his proofs the consideration of figures in three dimensions in order to arrive at properties of plane figures? This is today the distinctive feature of Monge's school in speculative geometry. (Chasles, 1837, p. 87)

And later:

this geometry, which is of a truly new kind, and which one rightly has called the school of Monge, [...] consists, as we just said, in introducing

10 Our statement should be qualified by saying that an intimation of such a possibility is given at the very end of the proof of the converse of Desargues' theorem on homological triangles given by Bosse. The proof concludes by remarking "And one can discuss their properties in the same way in the one figure as in the other, and so do without the solid figure, by using instead the figure in the plane" (cf. Gray \& Field, 1987, p. 164). Taton summarizes what is going on in Bosse's Proposition I as follows: "La marche suivie par Desargues, très claire et très rationelle, cadre parfaitement avec la nature de la question. Le théorème direct est démontré dans le cas de l'espace à trois dimensions par des propriétés d'incidence, puis dans le plan, par l'emploi répété du célèbre théorème de Ménélaüs, qui tenait déjà une place si importante dans le Brouillon project sur les coniques. La réciproque est ensuite démontrée suivant le même schéma appliqué à d'autres triangles. Dans une remarque finale, Desargues note que la figure formée par deux triangles perspectifs de l'espace se transforme, par projection cylindrique sur le plan d'un des triangles, en deux triangles perspectifs d'un même plan, suggérant ainsi la possibilité de démontrer le théorème du plan par les notions projectives de l'espace, et montrant en même temps l'intérêt de la projection cylindrique" (cf. Taton, 1951a, p. 203). 
in plane geometry considerations of solid geometry (Chasles, 1837,

p. 191; see similar claims also on p. 32 and p. 117)

While Desargues did not apparently use such considerations when proving the twodimensional version of his theorem on homological triangles, it seems that Poncelet was the first to do so. He was also the first to ascribe to Desargues the paternity of the theorem that we now call Desargues' theorem on homological triangles. ${ }^{11}$

We cannot of course give here a general account of how Monge and his school carried out such geometrical investigations (on this see Taton, 1951b; Belhoste, 1998; Belhoste \& Taton, 1989; Sakarovitch, 1989, p. 588) but we can at least give a simple but enlightening example of how Monge brought three-dimensional considerations to bear on problems in plane geometry. The theorem in question is found in Monge's lectures at the École Normale in 1795 (Monge, 1795, pp. 359-360). We will slightly simplify the statement and the proof to eliminate some trivial cases. Consider three circles with centers $A, B, C$ with different radii and whose centers are not collinear (as in Figure 3). Consider the common external tangents of each pair of circles and the respective points of intersection. The three points of intersection $D, E, F$, will be collinear (i.e., they lie on the same line).

Monge's proof proceeds immediately to embed the circles in space:

For if we consider the three spheres of which these three circles are the great circles and a plane that touches all three spheres externally, this plane will also touch the three conic surfaces circumscribed to the spheres considered pairwise and it will pass through their vertices. (Monge, 1795, p. 361)

11 Cf. Poncelet (1822, §168, pp. 89-90). The attribution of the result to Desargues is in the footnote on p. 89. He expressed the theorem, and his solid proof of it, as follows:

Deux triangles quelconques étant tellement disposés, sur un plan, que leurs sommets respectifs s'appuient, deux à deux, sur trois droites convergeant en un même point, les côtés opposés aux sommets qui se correspondent iront concourir, dans le même ordre, en trois point s situés en ligne droite; et réciproquement, si ces côtés concourent, deux à deux, en trois points situés en ligne droite, les droites qui joignent, dans le même ordre, les sommets correspondants des triangles iront converger en un même point.

Soient, par exemple, $A B C, A^{\prime} B^{\prime} C^{\prime}$ (fig. 22 et 23 ) deux triangles tellement disposés, que les droites $A A^{\prime}, B B^{\prime}, C C^{\prime}$ concourent en un même point $S$; les côtés $A B$ et $A^{\prime} B^{\prime}, B C$ et $B^{\prime} C^{\prime}, A C$ et $A^{\prime} C^{\prime}$ iront concourir respectivement aux trois points $I, K, L$ situés en ligne droite ; et réciproquement, si ces trois points sont sur une même droite, les trois droites $A A^{\prime}, B B^{\prime}, C C^{\prime}$ concourront en un même point $S$.

Cette relation nous offre le premier exemple de deux figures qui sont la projection ou perspective l'une de l'autre dans un plan (14), et il est bien digne de remarque que, pour l'établir, il n'est point indispensable d'avoir recours aux relations métriques des figures; car elle est évidente, à priori, pour le cas où les triangles sont dans l'espace, et elle le devient, par là même, pour celui où ils sont dans un plan, puisque l'une de ces figures peut toujours être considérée comme la projection de l'autre. Nous reviendrons, au reste, plus tard sur ces considérations, en les exposant dans toute leur généralité. 


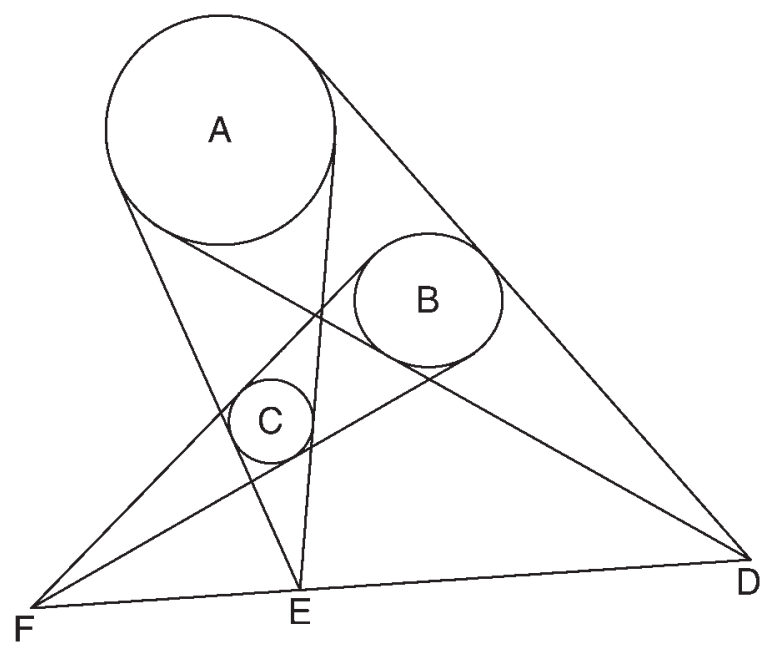

Fig. 3. Monge's theorem.

In other words, consider the three spheres as resting on the same plane $P_{1}$. Consider the plane $P_{2}$ tangent to the three spheres from above. Now any pair of spheres can be considered as being inscribed in a cone where the vertex of the cone is the point where the tangent to the circles from which the spheres originated intersect. Any plane that is externally tangent to two spheres will also be tangent to the cone. Since the radii of any two spheres are different, $P_{2}$ and $P_{1}$ intersect in a line $L$. Moreover, $L$ contains $D$ (as $D$ lies at the intersection of two planes tangent to the cone associated to the spheres with centers $A$ and $B$, i.e., $P_{2}$ and $P_{1}$ ), $E$ (as $E$ lies at the intersection of two planes tangent to the cone associated to the spheres with center $A$ and $C$, i.e., $P_{2}$ and $P_{1}$ ) and $F$ (as $F$ lies at the intersection of the planes tangent to the cone associated to the spheres with center $B$ and $C$, i.e., $P_{2}$ and $P_{1}$ ). Hence the three points are collinear.

Monge's theorem is intimately connected to Desargues' theorem although this observation might have escaped attention for a long time (see McCleary, 1982).

This concludes our sketch of uses of three-dimensional geometry for proving results in plane geometry. We have thereby indicated that the topic was of central interest to mathematical practice. A remarkable fact about the developments surveyed in this section is the lack of a debate on the foundational and methodological issues related to the exploitation of solid methods in plane geometry. We will now review how such methodological problems became an issue in the debate that opposed fusionists and antifusionists (separatists) in the late nineteenth and early twentieth centuries.

\$2. The debate on fusionism. The exploitation of stereometric considerations in plane geometry was to affect the elementary parts of the discipline as well. Already Gergonne in 1825 complained about the separation of planimetry and stereometry that was typical of Euclid and Legendre and advocated a joint development of the two branches of geometry. He remarked that the study of "extension" could be separated into two major areas, the one that studies metrical relations and the one that deals with positional (projective) relations. To determine the former requires calculation (in the form of analytic calculi) whereas projective relations are, in principle, independent of them. But the cost of eliminating 
the analytical reasoning from demonstrations of projective relations might require moving from plane to solid considerations:

But it can sometimes become necessary for this to move alternately from the geometry of the plane to that of space and then back again, as Monge and the geometers of his school have so often done and with so much success. It is therefore reasonable to wonder, on account of this, if our manner of dividing geometry into plane geometry and solid geometry is as natural and as exactly conforming to the essence of things as twenty centuries of habit have been able to persuade us. It still remains true at least that in renouncing this division one could succeed by having recourse, so to speak, to only simple intuition, in pushing forward in geometry beginners that the study of computation, presented at the start, too often only discourages, and that can be delivered later with much less repugnance, when their intelligence would be enlarged and strengthened by the study of a more or less prolonged series of properties of extension. (Gergonne, 1825-1826, pp. 209-210)

The above reflection by Gergonne highlights one interesting fact to which we will come back, namely that "purity" in the direction of eliminating metrical considerations from proofs of projective theorems might come at the cost of bringing considerations related to space in proofs of plane theorems. ${ }^{12}$ It will be only with the foundational work of Hilbert, foreshadowed by Peano and Pieri, that some of these claims would be put on a solid footing. ${ }^{13}$

Other geometers of the period, including Bellavitis and Cremona, pointed out the gain in simplicity afforded by moving to three-dimensional considerations:

From the outset I alternate without distinction theorems of plane geometry with those of solid geometry, for experience has taught me, and others had already observed this [Bellavitis, 1838, p. 247], that the stereometric considerations often well suggest the way of rendering easy and intuitive what in plane geometry would require a complicated and uneasy demonstration; moreover, they also sharpen the intellect and help develop geometric imagination which is an essential quality of the engineer, so that he might think figures in space without the aid of a diagram or of a model. (Cremona, 1873, p. xi)

Thus, mathematical practice spoke in favor of mixing considerations of plane and solid geometry on account of its success and the simplicity induced in the proofs. In addition, this was not only something that affected the advanced areas of the subject but rather even the writing of elements of projective geometry for high schools (such was the intended audience of Cremona's book).

While recent successes in mathematical practice obviously militated in favor of such admixture, the traditions of elementary geometry in Euclidian style spoke against it and some recent authors had also argued against appealing to spatial notions in plane geometry.

12 Unless otherwise stated, we use the term "space" to refer to three-dimensional space.

13 On projective geometry in the Italian school see Avellone et al. (2002) and Marchisotto \& Smith (2007); on Hilbert see Hallett \& Majer (2004). 
Bernard Bolzano in his revisionary Betrachtungen of 1804 argued against the use of spatial notions in definitions of plane notions:

Therefore we should regard as very artificial the definitions of the scholastic Occam, for solid, surface, line and point, according to which, solid is that kind of extension which cannot be the boundary of anything else, but surface is the boundary of a solid etc., because in order to conceive of only a point or a line they require in each case the idea of a solid.... However, it is obvious that we can perfectly well conceive of a surface, a line or a point, and that we do so without a solid which they bound. (Bolzano, 2005, p. 69)

And although he gave no example of such (at least we were not able to locate one in the Miscellanea Mathematica or in his published writings), one would expect him also to resist the use of solid geometry in proofs of plane theorems, for that would be consonant with the kind of purity programs Bolzano advocated throughout the essay. Unfortunately, Bolzano seems to have paid almost no attention to descriptive and projective geometry. Nonetheless, the issue of definitions of plane geometrical objects in terms of solid notions will also be part of the fusionist debate to be discussed below.

Despite the anticipations of authors such as Bretschneider (cf. Bretschneider, 1844) and Méray (cf. Méray, 1874), it was the Elementi di Geometria of R. de Paolis that brought the topic to the attention of the mathematical community. This was the first serious attempt to break the cleavage between plane and solid geometry in the developments of elementary geometry (and not only in projective geometry). De Paolis' goals are stated quite clearly in the preface to his book. He says:

In writing these Elements, I pursued two aims: To abandon the ancient separation between plane and solid geometry and to attempt to establish rigorously the fundamental truths of geometry and of the theories of equivalence, limits and measures. It is natural that I should have pursued a rigorous development and from the considerations that follow it will appear why I wanted to merge plane and solid geometry.

There is a broad analogy between certain plane figures and certain figures in space and if we refuse to study them together we decline the opportunity to learn everything that this analogy teaches us and we willingly end up in useless repetitions. Moreover, forcing ourselves to search the property of a line or of a surface without being able to use the entities lying outside this line or surface, we limit our forces and we intentionally renounce geometrical elements that would help us in simplifying the constructions or demonstrations. Indeed, how can one construct the middle point of a segment without going out of the segment itself? If one uses elements of a plane on which the segment lies, the proof is known and very easy. How can one construct an isosceles triangle with each of the two equal angles double in size the third angle without going out of its plane and applying the theory of equivalence or proportion theory? The triangle can be easily constructed without use of equivalence or proportion theory if we use geometrical entities that are outside its plane (p. 92). I could adduce other examples to show how much more one can simplify constructions and demonstrations by studying together plane and solid figures and I claim that if many such examples have not 
emerged in elementary geometry this is due to the constant and ancient division that has impeded their discovery. (De Paolis, 1884, pp. iii-iv)

Let us consider the theorem mentioned by de Paolis in his preface. It is a theorem of plane geometry which uses a construction that goes through space. The appeal to space is actually derivative from the use of a previous theorem (theorem 114) which reads as follows.

Theorem (114). When the vertices and the sides of two triangles correspond in such a way that the lines determined by the three pairs of corresponding vertices intersect in the same point and that the sides corresponding to the two pairs are parallel, then the sides of the third pair are also parallel.

Theorem 114 is an "affine" special case of Desargues' theorem, meaning that it is stated in terms of parallels. De Paolis' proof is of interest because it is a special case of the more general result that will be detailed later in the paper. His strategy is to solve the problem first for triangles lying in different planes and then to reduce the problem where the triangles are coplanar to the previous case.

In giving this proof we will need the following preliminary facts.

a) Two parallel planes are cut by a third plane along parallel lines.

b) If two planes passing through two given parallel lines meet, their intersection is a line parallel to the given parallel lines.

Now for the proof.

Consider two triangles $\triangle A B C$ and $\triangle A^{\prime} B^{\prime} C^{\prime}$. Assume that the lines $A A^{\prime}, B B^{\prime}, C C^{\prime}$ intersect in $P$ and that $A B, A^{\prime} B^{\prime}$ are parallel and so are $A C$ and $A^{\prime} C^{\prime}$. We need to show that $B C$ and $B^{\prime} C^{\prime}$ are also parallel.

Case I: the triangles are not in the same plane. In this case the theorem is immediate. Indeed, in this case the planes $A B C$ and $A^{\prime} B^{\prime} C^{\prime}$ are parallel. By a) a third plane (such as $P B C$ ) cuts the first two along parallel lines. Thus $B C$ and $B^{\prime} C^{\prime}$ are parallel for they are the intersection of the planes $A B C$ and $A^{\prime} B^{\prime} C^{\prime}$ with the plane $P B C$.

Case II: the triangles are in the same plane. On a line passing through $P$ we can select two points $Q, R$ not in the same plane as the triangles so that the pairs of lines $Q A, R A^{\prime}$; $Q B, R B^{\prime} ; Q C, R C^{\prime}$ meet in points $A_{1}, B_{1}, C_{1}$. The line $A_{1} B_{1}$ is on both planes $Q A B$ and $R A^{\prime} B^{\prime}$ and thus it is parallel, by b) to $A B$ and $A^{\prime} B^{\prime}$. By a similar argument, the line $A_{1} C_{1}$ is parallel to $A C$ and $A^{\prime} C^{\prime}$. But now we can look at the problem in space again. The pair of triangles $\triangle A B C, \triangle A_{1} B_{1} C_{1}$ and the pair $\triangle A^{\prime} B^{\prime} C^{\prime}, \triangle A_{1} B_{1} C_{1}$ satisfy the assumption of the theorem and lie on different planes. By case I, $B C$ and $B^{\prime} C^{\prime}$ are parallel to $B_{1} C_{1}$ and thus are parallel to each other.

De Paolis then gave, among other applications, the construction of the result mentioned in his preface by applying the plane version of the theorem we just presented.

It was the radical program of de Paolis' textbook, a program also pursued by Bassani and Lazzeri (cf. Lazzeri \& Bassani, 1898), that gave rise to a heated debate on the question of the "fusion" between plane and solid geometry. This debate was widely known. For instance, in the volume on geometry of his Elementary Mathematics from an Advanced Standpoint Klein refers to the debate remarking that, among other things, "Euclid is no 'fusionist' " (cf. Klein, 1953, p. 194; also see p. 2 for further discussion of fusionism).

In addition to articles in Italian published in the Bollettino of the association Mathesis, two articles in French — Candido (1899) and Loria (1905)—were responsible for bringing 


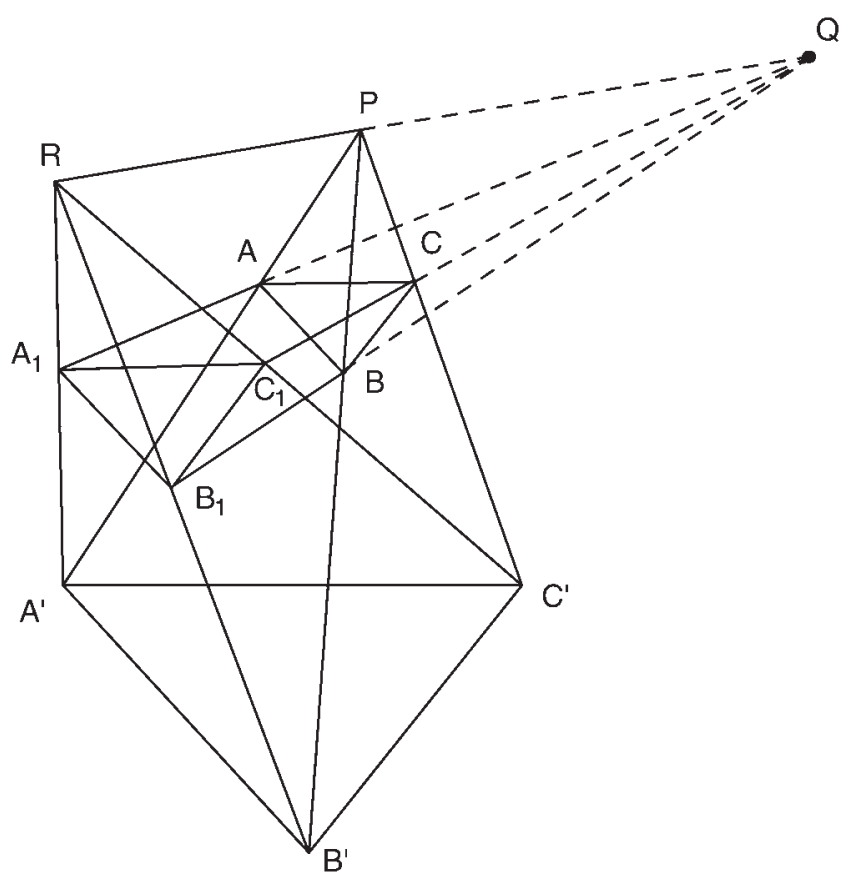

Fig. 4. De Paolis's proof.

the issue to the attention of the international community. We should point out from the outset that two major issues are subsumed under the umbrella term of "fusionism." One of them concerns the parallel development of plane and solid geometry to point out the analogies between the two areas (say between circles and spheres, angles, and diedra etc.). The second aspect is the one of greater interest to us, namely the use of stereometric considerations in the constructions, respectively proofs, of geometrical problems, respectively theorems. In any case a certain agreement was reached in defining "fusionism" as a "didactic method according to which from the very beginning one studies simultaneously similar topics in plane and solid geometry and then one applies in turn properties of one or the other in order to gain the utmost advantage from them" (Lazzeri, 1899, p. 117).

It will not be possible to recount blow by blow the discussion that ensued starting with the publication of de Paolis (1884) and which became the object of an open debate in the Bollettino dell'Associazione Mathesis and in the Periodico di Matematica on the question of whether it would be desirable to merge (or "fuse") together plane and solid geometry in teaching. This list would include Angeleri et al. (1904), Anonymous (1901), Bettazzi (1891), Candido (1899), de Amicis (1897-1898), Giudice (1899), Lazzeri (1899, 1904), Loria $(1899,1905)$, and Palatini (1897-1898, 1899). The debate has also not escaped the attention of historians of mathematics interested in the history of mathematical education (Bkouche, 1991, 1996, 2003; Giacardi \& Roero, 1996; Ulivi, 1977).

Three considerations were adduced in favor of fusionism. As summarized by Lazzeri (1899, pp. 118-119) they were:

1) Optimization of time obtained by treating together similar arguments of plane and solid geometry; 
2) Simplification of certain theorems and theories of plane geometry obtained by the help of stereometric considerations;

3) Better coordination of the study of mathematics with that of other subjects (physics, cosmography, crystallography) that are taught in technical high schools.

We will leave 1) and 3) to the side and focus on 2).

Issues related to 2) had already been raised in the long article de Amicis (1897-1898) "Pro Fusione." The article is a discussion of a number of opinions on the issue of fusionism submitted by several members of the association "Mathesis." 14

Not all of them were favorable to the idea of "fusion." For instance, Francesco Palatini (as reported by de Amicis) remarked that although he did not deny that certain proofs of plane geometry were simplified by moving to stereometric considerations, this did not mean that one might not be able with time to find proofs in plane geometry that were just as simple as the one obtained by moving to stereometric considerations. In addition, he also minimized the importance of fusion for metric geometry, observing that very few theorems that were not of a projective nature could be shown to be simplified in that setting: among the exceptions, the problem of constructing an isosceles triangle with each of the two equal angles twice as big as the remaining angle (this was the result mentioned by de Paolis in his preface). He also added that it was important for the students to understand that plane geometry has in itself all the resources for dealing with plane problems and does not need any "foreign" or "external" help. Finally, Palatini pointed out that the principle that "one should try to prove every proposition of elementary geometry without appeal to any theory on which it does not necessarily depend" had also been defended by the fusionists in the context of considerations of theories within plane geometry and he could not see why mathematicians such as de Paolis who were so "soft when it came to the distinctions between properties that belong to different theories within planimetry would want to enslave, so to speak, planimetry to stereometry" (cf. de Amicis, 1897-1898, p. 51).

We see that far from being limited to pedagogical considerations the above observations by Palatini lead naturally into foundational and methodological questions concerning "purity" and independence proofs.

Replying to Palatini's objections, de Amicis emphasized, to begin with, the advantage obtained in the logical treatment of theories brought about by de Paolis' approach. He mentioned, among other things, that the theory of the construction of regular polygons of $3,4,5,6,10$, and 15 sides that had been previously based on equivalence theory ${ }^{15}$ and proportion theory could now be developed before those theories and claimed that this represented progress in the logical order of the treatment, in addition to scientific and

14 On the history of the association Mathesis see Giacardi \& Roero (1996) and Bolondi (2002).

15 The theory of equivalence studies when two polygonal figures in the plane have the same area. The relationship of equivalence can be set out axiomatically (this development originates with de Zolt in 1880) by stating principles to the effect that two figures are equivalent if and only if they are sums of parts that are pairwise equal (congruent). There are also further extensions of the theory to nonpolygonal plane figures and to solid geometry. For a clear historical and technical presentation of the theory of equivalence as it presented itself in 1900 see the informative article Amaldi (1900, pp. 103-142). Amaldi touches on issues related to the development of the theory independently of proportion theory on p. 115. 
pedagogical progress. Other examples of simplification effected by bringing in stereometric considerations were adduced, such as the emancipation of the theory of equivalence from proportion theory, the emancipation of the theory of radical axes from both of the previous theories, and finally the development of the theory of homothetic figures from that of proportion theory.

Against Palatini's conjecture that simple proofs of plane results by planimetric considerations could still be found (thereby making the recourse to stereometric considerations less appealing), de Amicis retorted by pointing out that this would be unlikely since plane geometry had been studied for more than 22 centuries and if such easy proofs were available they would have been discovered by then. By contrast, stereometric considerations in plane geometry were new and one had to expect that the number of successful applications would increase.

Of interest here is also a passage from Veronese cited by de Amicis, for it brings to the fore also considerations related to hyperspaces and the analogous problem of using spaces of higher dimensions in proving results for spaces of lower dimension. ${ }^{16}$

Veronese was however negative on the usefulness of stereometric considerations in plane geometry when it came to metric properties:

Euclidean elementary geometry is mainly metrical in nature and it is based on the concept of equality.... In my geometrical works I have advocated not only the fusion of ordinary space geometry [solid geometry] with that of plane geometry, but also that of higher spaces with that of lower spaces, for indeed the study of projective properties of the latter spaces finds in the use of the former spaces the highest and most complete development; nonetheless, I must recognize that solid geometry is in this sense of little help to ordinary plane geometry because the latter is, as I said, essentially of metrical nature. (Veronese \& Cazzaniga, 1897, Introduction)

In addition de Amicis also pointed out that Peano had clearly seen that Desargues' theorem on homological triangles in the plane was a theorem of solid geometry (he referred to Peano, 1894, p. 73; whether Peano, 1891, already makes this point is not clear ${ }^{17}$ ).

De Amicis then argued that even the "separatists" were implicitly committed to spatial notions, for the notion of equality typical of Euclidean geometry, namely that based on superposition (and congruency), required motion and showing congruency in some cases required flipping a figure outside its own plane. And this would be an impossibility for

16 This was a topic of debate between Segre (cf. Segre, 1891), Veronese and Peano; see Manara \& Spoglianti (1977).

17 Peano's (1891) text is of interest first of all for his "conventional" distinction between plane and solid geometry, which he relativizes to the choice of axioms. In addition, Peano seems to hold what we might call a "logical" notion of content, according to which a statement turns out to be plane or solid not depending on its informal content but rather on whether it can be derived from the conventionally established system of axioms. (We will discuss related positions by Carnap and Hallett later in the article.) Secondly, the text does seem to anticipate the claim of Peano (1894), for it says that Desargues' theorem on homological triangles is a theorem of solid geometry. However, it does not explicitly specify whether the plane version of Desargues' theorem is also under consideration at this stage, and thus it is not clear to us, although we do consider it quite likely, that Peano is already making the claim that he would make in 1894. 
a "plane" purist who, using a culinary metaphor, "could not flip the omelette."18 As a consequence, many theorems could only be proved by making special additional postulates. In short, the separatists who will insist on the separation between plane and solid geometry will have to pay a high cost by being forced to make all kinds of extra postulates to be able to implement the desired separation (cf. de Amicis, 1897-1898, p. 62). The "separatist" geometers who relied on the Euclidean notion of equality were thus "fusionists" without knowing it. Finally he asked: "Perchè questo orrore dello spazio? [Why this fear of space?]" (p. 65), while its use could be so beneficial and it is anyhow the most natural concept?

Still in reply to Palatini concerning the issue of rendering theories independent of other theories which are not necessarily connected to it, de Amicis proposed an interesting example of a theorem (closely related to de Paolis' theorem 114) whose statement only involved parallelism and equality. This clearly illustrated that fusionism was motivated by such methodological considerations. De Amicis gave three proofs of the same result. The first two used crucially proportion theory and the theory of equivalence while the third avoided such uses by means of stereometric considerations. With the latter demonstration, according to de Amicis, one does not go out of the theory of parallels and equality, which are the notions mentioned in the statement of the theorem, even if one has to go through space, whereas with the former two proofs one needs to appeal to the theory of equivalence and proportion theory which are not connected to the problem.

Lazzeri (1899) also focuses on the second of three considerations generally adduced in favor of fusionism. Lazzeri, like de Amicis, was a strenuous defender of fusionism. He emphasized that the use of stereometry had given the means to render the theory

18 Let us mention as an aside that the point made above was already made by Möbius in his Der barycentrische Calcul (cf. Möbius, 1827, part 2, chapt. 1):

It seems remarkable that solid figures can have equality and similarity without having coincidence, while always, on the contrary, with figures in a plane or system of points on a line equality and similarity are bound with coincidence. The reason may be looked for in this, that beyond the solid space of three dimensions there is no other, none of four dimensions. If there were no solid space, but all space relations were contained in a single plane, then it would be even as little possible to bring into coincidence two equal and similar triangles in which corresponding vertices lie in opposite orders. Only in this way can we accomplish this, namely by letting one triangle make a half revolution around one of its sides or some other line in its plane, until it comes into the plane again. Then with it and the other triangle will the order of the corresponding vertices be the same, and it can be made to coincide with the other by a movement in the plane without further assistance from solid space. (Translation from Van Cleve \& Frederick, 1991, p. 40)

A similar point is made in Wittgenstein's Tractatus 6.36111: "Kant's problem about the right hand and the left hand, which cannot be made to coincide exists even in two dimensions." There are connections here with the development of the concept of symmetry. See for instance Hon \& Goldstein (2008, p. 215 and pp. 234-235). It was Hilbert who investigated foundationally this kind of phenomenon by looking at the relationship between weakenings of the Triangle Congruence Axiom (TCA) and the provability/unprovability of the Isosceles Triangle Theorem (Euclid I.5). In this context the TCA guarantees the validity of the side-angle-side criterion for triangle congruence. In essence it legitimizes the move according to which, any one of two triangles on the same plane satisfying the conditions of the TCA but which do not have the same orientation can be 'flipped' in space so as to overlap with the other. For an exposition of this fascinating bit of foundational work see Hallett (2008, pp. 229-239). 
of equivalence independent of proportion theory. He pointed out that in his book with Bassani such stereometric considerations were also used in giving theorems on similarity without assuming proportion theory. The important thing about such examples is that the simplification does not affect single theorems but rather entire theories. In this way he rebutted the objections of the separatists who insisted that there were very few theorems for which one could claim an advantage by introducing stereometric considerations (division of the right angle into five parts; the already mentioned theorem on isosceles triangles). But in an interesting reply to an objection raised by his colleague Retali, Lazzeri also touched upon the issue of the relation between hyperspaces of $n$ dimensions and spaces of lower dimension. The objection by Retali was to the effect that Riemann had shown how in the study of a space of $n-1$ dimensions one can abstract from the properties of a space on $n$ dimensions in which it is embedded, whereas lower-dimensional spaces are used when developing the theory of higher-dimensional spaces. Lazzeri granted that this was true to the extent that we need to rely on properties of lower dimensional spaces in order to state the postulates for higher dimensions. However,

This does not prove that-after having established the fundamental properties of a hyperspace-one cannot obtain from its more elementary properties, in a simple and expedited way, other more complicated properties of figures belonging to spaces with a lower number of dimensions. The examples that one could adduce in this connection-obtained from higher geometry to show how from the properties of an hyperspace one can deduce other properties of spaces with a lower number of dimensions - are innumerable. It will be enough to mention the plane representation of surfaces, by means of which one can from the properties of surfaces obtain properties of curves and vice versa; the representations of bundles of lines or spheres in ordinary space, the study of Pascal's hexagon deduced from the figure of six complexes of six connected [entities] which are in involution in pairs, and many others. (cf. Lazzeri, 1899, p. 123)

Palatini in his reply tackled one of the examples that had been paraded by the fusionists in their favor (theorem 1 on p. 303 of de Paolis' Treatise); we do not need to state it for our purposes but let us call it theorem A. From it follows the theorem, let us call it B, to the effect that given two triangles with their angles respectively equal, the sides of the first are proportional to the sides of the second. He objected that while de Paolis' proof of A avoided the use of proportions, it required both appeals to the theory of equivalence and to stereometry. But if B is inferred from A, as one would normally do, then B would be dependent on the theory of equivalence and stereometry, neither one of which was necessarily connected to the statement of the theorem. Thus, "one frees a slave from one master in order to render a free citizen slave of two masters" (Palatini, 1899, p. 207). In addition, he also offered a new proof by planimetric considerations (which did not depend on the theory of equivalence or proportion theory but used the Archimedean axiom) of a result from which, he claimed, all of the theorems that were offered by the fusionists as showing the advantage of using stereometry depended and could easily be proven. This is the theorem that played a central role in de Paolis' treatise and that we have proved above: "If two triangles correspond to each other in such a way that the lines connecting their respective vertices meet in a point and that two sides of one triangle are parallel to the corresponding ones of the second, then the remaining two corresponding sides are parallel." With this example Palatini claimed to have shown that despite the old pedigree of 
planimetry new simple proofs could always be found and that stereometric considerations could be avoided.

This article was the last in which serious methodological questions were addressed. Lazzeri (1904) and Angeleri et al. (1904) are unfortunately only polemical and without much value.

In conclusion, we have seen how the fusionist-separatist debate brought to the fore issues of purity both for single theorems as well as for entire mathematical theories. However, such discussions could not be properly settled without the kind of work that mathematicians such as Peano and Hilbert were already addressing and that required the finer use of logical and axiomatic tools. In addition, several philosophical problems were left without proper analysis in the discussion: when is a solution to a problem (or a proof of a theorem) "foreign" to its content? How can we articulate this notion of content analytically? Answers to such questions are essential if a debate like the one we have covered in this section can have any hope of moving beyond subjective opinions. In this case only a combination of foundational work and the development of the appropriate analytical tools for the explication of such notions as "purity" and "content" can hope to indicate the way for progress. It would of course be beyond the scope of a single paper to try to address the full variety of theories that had come up for discussion in the fusionist-separatist debate (projective, metric, theory of equivalence, proportion theory, etc.). Rather, we will focus on a single, but extremely rich, case study, namely the role of Desargues' theorem on homological triangles in the foundational debates on projective geometry. This case study will provide us with a way to bring into sharper focus the issues related to "purity" and "content" raised in the fusionist debate.

§3. Desargues' theorem: a case study. In the early nineteenth century geometers set out to develop the foundations of projective geometry, independently of Euclidean geometry. Some, for instance Möbius (cf. Möbius, 1827) and Plücker (cf. Plücker, 1830), sought to develop an analytic projective geometry, analogous to Cartesian analytic geometry for Euclidean geometry. They developed systems of "homogeneous" coordinates for projective geometry analogous to Cartesian coordinates for Euclidean geometry. ${ }^{19}$ This new analytic geometry was quite powerful, allowing simpler proofs of geometrical theorems than were otherwise available and enabling rapid progress in the discovery and proof of new results. ${ }^{20}$ Others, for instance Steiner (cf. Steiner, 1832), sought a coordinate-free development of projective geometry that had the same power as the new analytic projective geometry.

19 Homogeneous coordinates permit the analytic treatment of points and lines in the projective plane as follows. In a "homogeneous" equation all the terms are of the same degree. The homogeneous equation $a X+b Y+c Z=0$ may be associated with the linear equation $a x+b y+z=0$ in the following way: given a triple $(X, Y, Z)$ with $Z \neq 0$ satisfying $a X+b Y+c Z=0$, the pair $\left(\frac{X}{Z}, \frac{Y}{Z}\right)$ satisfies $a x+b y+z=0$. We may use such triples $(X, Y, Z)$ to represent the point $\left(\frac{X}{Z}, \frac{Y}{Z}\right)$ in the Euclidean plane. Triples $(X, Y, Z)$ with $Z=0$ may be used to represent "ideal" points at infinity in the projective plane, for which there is no Euclidean correspondent; this is a convenient representation since the Euclidean pair $\left(\frac{X}{Z}, \frac{Y}{Z}\right)$ involves division by zero when $Z=0$. This coordinatization also provides for equations for all the projective lines, and can provide equations for algebraic curves in the projective plane as well. For more details, cf. Seidenberg (1962, pp. 35-38, 172-174).

20 The precise nature of these improvements is quite complex and will be addressed in detail elsewhere. 
In this work these geometers freely used metric considerations. They appealed either to the Euclidean distance metric, or to principles of proportionality or congruence, for instance using what Chasles called the "anharmonic ratio" and Clifford called the "crossratio" of four collinear points. ${ }^{21}$ Beginning with his Geometrie der Lage (cf. von Staudt, 1847), von Staudt sought to eliminate these metric considerations from projective geometry on what can justly be characterized as appealing to considerations of "independence": "I have tried in this work to make the geometry of position into an independent science [selbstständigen Wissenschaft] that does not require measurement [Messen]" (cf. von Staudt, 1847 , p. iii). As justification of this approach he explained that "geometric instruction must proceed from general considerations" and that his approach differs from metric investigations that "move too soon to the particular, namely the congruence and similarity of triangles, and therefore do not establish enough concepts in proper generality." The idea seems to be that projective geometry is the most "general" or "basic" geometry, and that to study geometry with metric assumptions in force is to limit the study's generality to only a special case of this basic geometry; and that one should seek to develop geometrical concepts in this more general or basic way. He did not indicate why he judged this type of development to be desirable, though.

Though there were gaps concerning continuity that were later filled by others, von Staudt's work yielded a means of defining both projective coordinates and cross-ratio by purely projective means. The key to his accomplishment was his "quadrilateral construction," which provides a way, given any three collinear points, to find uniquely a fourth point on that line by means of a construction that we explain immediately; it is then said that the four points together form a "harmonic range." The construction proceeds as follows (an example is depicted in Figure 5): suppose $A, B$, and $C$ are three collinear points, and that $E$ is a point not collinear with these three. Let $C F G$ be a line through $C$ not identical with $A B C$ that does not pass through $E$, with $F$ the point where $C F G$ passes through the line $B E$ and $G$ the point where $C F G$ passes through the line $A E$. Let $H$ be the point where the line $A F$ intersects the line $B G$. Then $D$ is the point where the line $E H$ intersects $A B C$. In this case, $D$ is said to be the "harmonic conjugate" of $C$ with respect to $A$ and $B$ (cf. von Staudt, 1847, §93). The quadrilateral construction thus constructs this fourth point $D$ of a harmonic range consisting of $A, B, C$, and $D$.

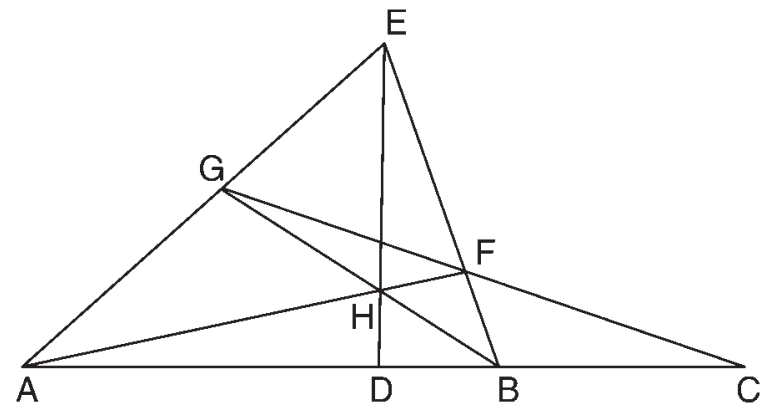

Fig. 5. von Staudt's quadrilateral construction.

21 The "cross-ratio" of four collinear points $A, B, C$, and $D$ is the quantity $\frac{C A}{C B} / \frac{D A}{D B}$, where the line in question is supposed to have an orientation so that this quantity can be negative or positive depending on the order of the points. Since Desargues cross-ratio had been known to be projectively invariant. 


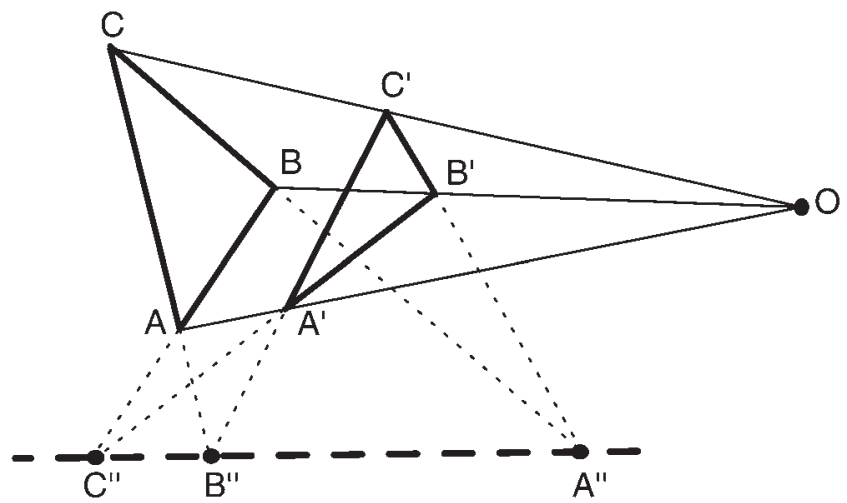

Fig. 6. Desargues' theorem.

It can be shown that four points in a harmonic range have cross-ratio -1 , and this turns out to be critical for the quadrilateral construction's usefulness in replacing appeals to cross-ratio. Indeed, this gives a way of carrying out the quadrilateral construction, and showing the uniqueness of the fourth harmonic point, by appeal to metric considerations. Following his aim of purifying projective geometry of metric considerations, von Staudt instead proved the uniqueness of the fourth harmonic point by purely projective means, in particular by appealing to Desargues' theorem, to which we now turn our attention.

There are several ways of formulating what is known as "Desargues' theorem," but its most common formulation is the following: if $\triangle A B C$ and $\triangle A^{\prime} B^{\prime} C^{\prime}$ are two triangles lying in the same plane and such that the lines $A A^{\prime}, B B^{\prime}$, and $C C^{\prime}$ intersect at a point, then the intersections $C^{\prime \prime}, A^{\prime \prime}$, and $B^{\prime \prime}$ of the corresponding sides of $\triangle A B C$ and $\triangle A^{\prime} B^{\prime} C^{\prime}$ - that is, the intersections of $A B$ and $A^{\prime} B^{\prime}, B C$, and $B^{\prime} C^{\prime}$, and $A C$ and $A^{\prime} C^{\prime}$-are collinear. (See Figure 6 for an example of such a configuration.) Since two triangles are said to be "perspective from a point" if the lines through their corresponding vertices are coincident in a single point, and to be "perspective from a line" if the intersections of their corresponding sides are collinear, this result can be stated concisely as saying that if two triangles in the plane are perspective from a point, then they are perspective from a line. If the result so formulated is true, its converse is also true, and so geometers sometimes choose its converse formulation instead.

Both of these formulations are "projective," concerning only the incidence and intersection of points and lines, where the points-if we avail ourselves of nonprojective terminology_may be either the "real" points of familiar geometric experience or "ideal" points at infinity where parallel lines meet. There are also "affine" formulations of Desargues' theorem, involving parallels and avoiding points at infinity. We have seen one when discussing de Paolis above; Hilbert's formulation (of the converse) is another such formulation (cf. Hilbert, 1899, theorem 32): "when two triangles are so situated in a plane that their homologous sides are respectively parallel, then the lines joining the homologous vertices pass through one and the same point, or are parallel to one another."

Desargues' theorem can be proved easily using metric methods, in particular by way of Menelaus' theorem (which requires congruency for its proof), as Desargues had done (cf. Coxeter \& Greitzer, 1967, p. 71). ${ }^{22}$ Such proofs are not satisfactory for von Staudt's

22 For a study of Menelaus' theorem in axiomatic geometry, see Guggenheimer (1974), and for the weakest congruence axioms strong enough to imply Desargues' theorem, see Sperner (1954). 
purpose of purifying projective geometry from metric considerations. In von Staudt's time purely projective proofs were also known, though, and von Staudt gave such a proof (cf. von Staudt, 1847, §90). Strikingly, his proof draws on considerations from solid geometry (ibid., §87), despite the fact that Desargues' theorem concerns just triangles in the plane. In giving a solid proof von Staudt was following previous work relating Desargues' theorem stated for triangles in the plane with its solid analogue for triangles lying in different planes. This solid analogue states that if $\triangle A B C$ and $\triangle A^{\prime} B^{\prime} C^{\prime}$ are two triangles lying in different planes and such that the lines $A A^{\prime}, B B^{\prime}$, and $C C^{\prime}$ intersect at a point, then the intersections of their corresponding sides are collinear. The solid Desargues theorem had been noted for its obviousness; Poncelet for instance had called the solid version "évidente, à priori" (Poncelet, 1822, §168, p. 89). It can be proved projectively as follows (see Figure 7 for illustration). Suppose $\triangle A B C$ and $\triangle A^{\prime} B^{\prime} C^{\prime}$ are two triangles lying in different planes and such that the lines $A A^{\prime}, B B^{\prime}$, and $C C^{\prime}$ intersect at a point $O$. The intersections of $A B$ and $A^{\prime} B^{\prime}, B C$, and $B^{\prime} C^{\prime}$, and $A C$ and $A^{\prime} C^{\prime}$, each lie in both of the two planes. Since we are working in projective space the two planes intersect, and their intersection is a line. Therefore the intersections of the corresponding sides of $\triangle A B C$ and $\triangle A^{\prime} B^{\prime} C^{\prime}$ are collinear.

In Gergonne (1825-1826, p. 217), Gergonne, after remarking on the importance of solid methods in planar geometry (quoted earlier on p. 303), states Desargues' theorem in both solid and planar versions, proves the solid version, and argues that this solid proof establishes as a derivative case the planar Desargues theorem. To see how this goes, suppose that there is a point $S$ outside the plane in which $\triangle A B C$ and $\triangle A^{\prime} B^{\prime} C^{\prime}$ lie (see Figure 8). On the line $S B$ let $B_{0}$ be a point, and let $A B_{0} C$ be a plane in which $A C$ lies and which passes through $B_{0}$. The triangle $\triangle A B_{0} C$ lies in this plane. Since both $O B_{0}$ and $S B^{\prime}$ lie in the plane $O B S$, they intersect in this plane; let $B_{0}^{\prime}$ be that point of intersection. Then $A^{\prime} B_{0}^{\prime} C^{\prime}$ is a plane in which the triangle $\triangle A^{\prime} B_{0}^{\prime} C^{\prime}$ lies. Then $\triangle A B C$ and $\triangle A^{\prime} B^{\prime} C^{\prime}$ are planar projections from $S$ of $\triangle A B_{0} C$ and $\triangle A^{\prime} B_{0}^{\prime} C^{\prime}$, respectively. Furthermore, $\triangle A B_{0} C$ and $\triangle A^{\prime} B_{0}^{\prime} C^{\prime}$ are two triangles lying in different planes for which the lines $A A^{\prime}, C C^{\prime}$, and $B_{0} B_{0}^{\prime}$ connecting their corresponding vertices intersect at $O$, and so satisfy the antecedent of the solid Desargues theorem. The line in which their two planes intersect can be

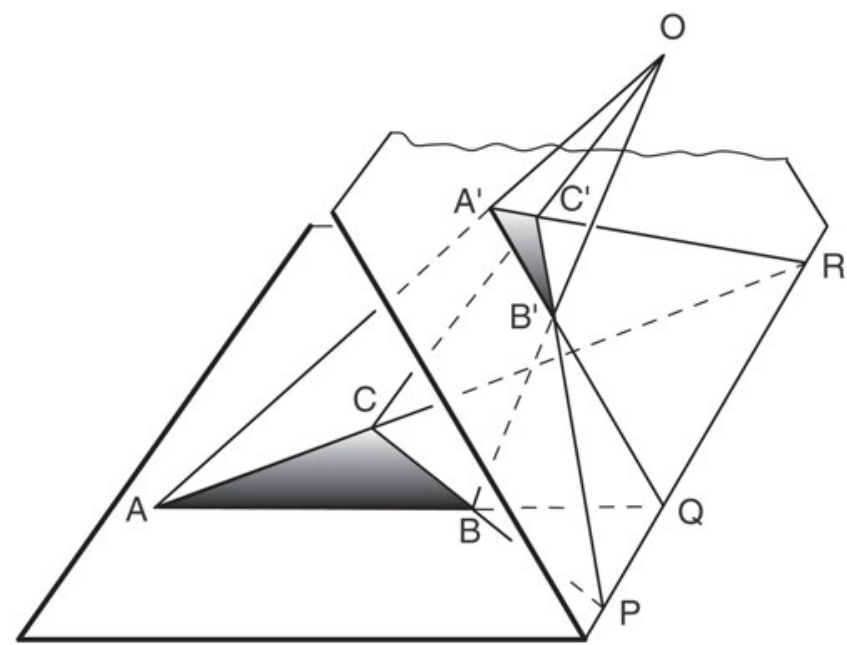

Fig. 7. A proof of Desargues' theorem for triangles in different planes (adapted from Courant \& Robbins, 1996, p. 171) . 


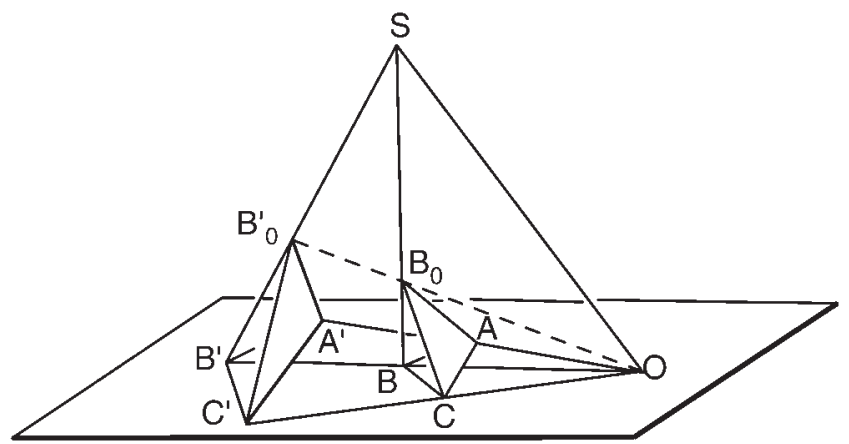

Fig. 8. A projective proof of the planar Desargues theorem, using solid methods (adapted from Hilbert \& Cohn-Vossen, 1952, p. 122).

projected from $S$ onto the plane in which the planar triangles $\triangle A B C$ and $\triangle A^{\prime} B^{\prime} C^{\prime}$ lie. Then the intersections of corresponding sides of $\triangle A B C$ and $\triangle A^{\prime} B^{\prime} C^{\prime}$ intersect on this projected line, as we were trying to prove.

Thus Desargues' theorem in the plane is proved by solid methods. No projective proof using purely planar methods was known in the nineteenth century, however. This peculiar state of affairs was noticed by nineteenth-century geometers, as we have already seen in Sections 1.3 and 2. In the second half of the century several geometers investigated why this state of affairs held, that is, whether there could be a planar projective proof of Desargues' theorem. Among these are Klein, Peano, and Hilbert. We will now consider their observations in turn.

3.1. Klein. Klein (1873) sought to develop the foundations of projective geometry, in particular the development of projective coordinates as von Staudt had carried out, without following von Staudt in using the parallel axiom. Von Staudt drew on Desargues' theorem in developing these coordinates, as we explained earlier, and not only did he draw on solid methods in proving Desargues' theorem, his development of projective geometry consistently fused solid and planar methods together. His coordinates were, as Klein noted, coordinates for projective space, not just for the plane. Klein showed how these coordinates allowed surfaces in space meeting certain basic conditions to be represented by linear equations. This was desirable because it enabled analytic computations concerning these surfaces to be more easily carried out. As he saw it, a spatial analogue of the projective geometry of planes and lines is valid for these surfaces and curves on those surfaces. ${ }^{23}$

Klein asked whether such a development could be carried out for the plane alone; that is, whether von Staudt's methods could generate coordinates for just the plane, considered alone rather than as an element of space, such that planar curves meeting basic conditions similar to those in the case of surfaces would be representable by linear equations. If this were possible, then it would indicate that the projective geometry of the plane could be developed without treating the plane as an element of, or "embedded in," space. He observed that it could not, as a consequence of a result of Beltrami's. Beltrami stated his result as follows:

23 In his words (cf. Klein, 1873, p. 135), "Für ein solches System von Flächen und Curven gilt die projectivische Geometrie in demselben Sinne wie gemäss den gewöhnlichen Vorstellungen für das System der Ebenen und Geraden in einem beliebig begrenzten Raume." 
The only surfaces susceptible of being represented on a plane, so that each point corresponds to a point [on the plane] and each geodesic to a straight line, are those whose curvature is everywhere constant (positive, negative or zero). (cf. Beltrami, 1865, p. 279 in the reprint in Opere matematiche)

That is, a surface has constant curvature if and only if it can be mapped to a plane so that the geodesics of that surface (roughly, the curves tracking the shortest distance between points on that surface) are mapped to straight lines in that plane. Beltrami observed that such mappings are important in the construction of planar maps of the earth, where a surface of constant curvature (the idealized sphere of the earth) is mapped by central projection to the plane. Figure 9 illustrates such a mapping, with $A B$ a great circle on the sphere, and $A^{\prime} B^{\prime}$ its image in the plane.

Actually, Beltrami's result only applies to Riemannian surfaces, which are "smooth" surfaces (in the sense of being sufficiently differentiable everywhere) endowed with a smoothly varying Riemannian metric (cf. Busemann, 1955, for a more technical definition). In the nineteenth century only surfaces endowed with smooth metrics were considered, so Beltrami's work can be understood as implicitly restricted to Riemannian surfaces. Klein's remarks are thus also restricted to Riemannian surfaces (hence we continue by writing "surface" for Riemannian surface unless we have explicit reason to think such a restriction is not in place).

Klein understood Beltrami's theorem as asserting that one cannot represent a surface of nonconstant curvature on the plane such that all the geodesics of that surface are represented by straight lines in the plane. The result applies to the Euclidean plane and even to the projective plane. So Klein seems to have concluded from Beltrami's theorem that surfaces whose geodesics have linear equations in projective coordinates (satisfying the aforementioned basic conditions), and thus whose planar representations "behave" like projective straight lines, must have constant curvature. But then planes that represent surfaces of nonconstant curvature have lines that are not all representable by linear equations and hence do not all behave like projective straight lines. Klein thus recognized a fundamental difference between the projective geometry of space, including planes and lines in those planes as elements, and the projective geometry of planes treated independently from space rather than as embedded in space. As he put it:

Thus, one must not, as it has happened since, regard it as a trick [Kunstgriff] by von Staudt when he also considered the stereometric relationships of the plane for the foundation [Begründung] of projective geometry. His starting point corresponds completely to the nature of the

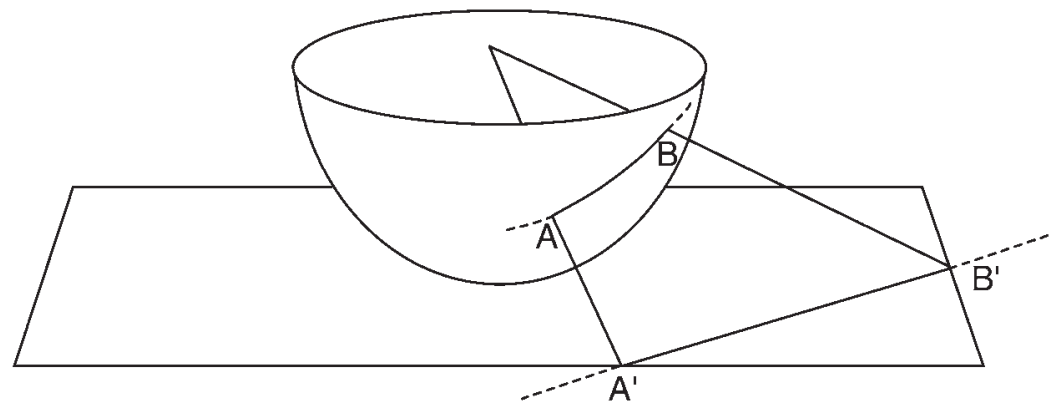

Fig. 9. Central projection of a great circle on a sphere to the plane. 
matter. If, following von Staudt, one excludes the consideration of metric relations, then projective geometry holds for the straight lines in a plane because plane and line can be conceived as elements of a spatial system. (cf. Klein, 1873, p. 136)

In this striking passage, Klein asserts that von Staudt's fusionist treatment of the plane and space "corresponds completely to the nature of the matter," because a purely projective treatment of the plane, without taking it as embedded in space, will fail on account of Beltrami's theorem. Planes not embedded in space may include geodesics that fail to behave like projective straight lines in the sense that they are not representable by linear equations in projective coordinates, since such planes may have nonconstant curvature. The geodesics of planes embedded in space do behave like projective straight lines, though (provided that their geodesics satisfy the aforementioned basic conditions). In this way the projective geometries of space and planes not taken as embedded in space differ.

Klein's concern was with finding a projective geometry that supported the right kind of coordinatization, and not explicitly with Desargues' theorem. Therefore he does not draw any explicit connection between this observation and the possible failure of Desargues' theorem in a purely planar setting. But what he says suggests this connection, since the key to von Staudt's coordinatization of projective space was Desargues' theorem.

This connection was picked up soon thereafter, though. In an influential 1891 lecture (published a year later as Wiener, 1892) Wiener gave Desargues' theorem as an example of a theorem of planar projective geometry, emphasizing that it is a theorem concerning the incidence and intersection of lines and points (p. 46). While noting that it can be proved by spatial means he remarked, without proof, that it cannot be proved by purely planar incidence and intersection considerations, observing that "this area of geometry is not self-contained" (p. 47). Peano and Hilbert took up this metamathematical question shortly thereafter, and to this work we now turn.

3.2. Peano. During the 1890 s the systematic study of projective geometry was in full swing in Italy (providing a crucial background to the fusionism debate discussed in Section $\S 2)$. For instance Pieri provided several different axiomatizations of projective geometry, including solid axioms, typically noting which of his axioms suffice for von Staudt's spatial proof of Desargues' theorem. But it was Pieri's fellow countryman Peano who seems to have been the first to find a model of the plane in which Desargues' theorem fails, in an 1894 essay (Peano, 1894). Priority for this result is typically given to Hilbert; for example, the claim is made in Hallett (2008, p. 225): "Hilbert's model specifies the first example of a non-Desarguesian geometry." On the contrary, we concur with Whitehead (1906, p. 11), who attributes the result to "Peano, and subsequently Hilbert," citing Peano (1894) and in particular p. 73, which we discuss shortly. Whitehead does not substantiate this claim with a discussion of Peano's work, however. Accordingly, we will attempt to do so.

While it is true that Peano's setting is not clearly projective- - he follows Pasch in taking as a primitive notion the ternary relation of betweenness, which is not projectively invariant (on this, see Section §6) — his proofs bring to light both spatial and metrical issues that will concern us for the remainder of the paper.

Peano firstly gave postulates I to XIV evidently concerning planar geometry, and secondly introduced postulate XV in a section entitled "solid geometry." 24 Peano stated

24 Note, however, that Peano's expression of Postulate $\mathrm{X}$ in his formal language differs from its subsequent informal expression. In the last clause of the formal version, it says that $a$ is between 
it as follows: "Given a plane $h$, we can find a point a outside of it." Shortly thereafter, Peano turned to Desargues' theorem, writing as follows:

The theorem on homological triangles in the plane [Desargues' theorem], is however a consequence of postulate $\mathrm{XV}$, and thus it is a theorem of solid geometry. That it is not a consequence of the preceding postulates follows from the following consideration. If by $p$ we mean the points of a surface and with $c \in a b$ we mean that the point $c$ lies on the geodesic arc that joins the points $a$ and $b$, then all the postulates I to XIV are satisfied but the proposition on homological triangles is not always true. This proposition, however, still holds for surfaces with constant curvature. (cf. Peano, 1894, p. 73)

Peano thus proposed doing planar geometry on surfaces other than the standard "flat" plane, by taking on those surfaces the "straight lines" between two points to be the geodesics between those two points. As with Klein, we may suppose that Peano's intended surfaces are Riemannian. Peano claimed that for surfaces satisfying his postulates I to XIV, Desargues' theorem holds for triangles when the surface has constant curvature, and that it may fail when the surface has nonconstant curvature. That is, he claimed to have identified a model in which postulates I through XIV are satisfied but Desargues' theorem is false, without specifying the model explicitly. His claim is simply that surfaces of nonconstant curvature satisfying his first fourteen, purely planar, postulates need not satisfy Desargues' theorem. From this he concluded that Desargues' theorem is not a consequence of his planar postulates, and hence requires solid postulates for proof in his axiomatic framework.

To judge Peano's priority in finding a non-Desarguesian plane, it would be good to know what models he might have had in mind, and to understand why Desargues' theorem fails in these models. Thus we will consider specific elementary surfaces of nonconstant curvature, the ellipsoids, and show the failure of Desargues' theorem on this surface. We will do so in terms available to geometers of Peano's ilk at the time. It is thus plausible that he was thinking in this way.

The construction of this model goes as follows. ${ }^{25}$ We begin with a disk $D$ in a Euclidean plane $P$ embedded in a Euclidean space of three dimensions. Let $O$ denote the center of $D$ and let $C$ denote the circle that is the boundary of $D$. We consider ellipsoids generated by revolving an ellipse around one of its axes, this axis being perpendicular to $P$ at $O$, such that the generated surfaces have center $O$ and intersect $P$ along $C$. Let $h$ denote the length of the segment cut by the ellipsoid on its axis of revolution, from $O$ to the edge of the ellipsoid. (Figure 10 depicts such an ellipsoid.) We will consider in particular ellipsoids with very small values of $h$, that is, very "flat" ones. When $h$ approaches 0 , the ellipsoid converges to the disk $D$ covered twice (by each "side" of the ellipsoid).

Continue by projecting all points on the ellipsoids onto the plane $P$ in which $D$ lies, by a cylindrical projection perpendicular to $P$. Consider the images of the geodesics of these ellipsoids by this projection. When we vary $h$, the images of these geodesics vary. At the limit, the configuration of all the geodesics on the ellipsoid converges to the configuration of Euclidean lines inside the disk $D$. In this limiting case, when a line in $D$ reaches the

$b$ and $c$. But in the informal description, it says that $d$ is between $b$ and $c$. It is evident that the informal version expresses what Peano intended.

25 We are in debt to Patrick Popescu-Pampu for this construction. 


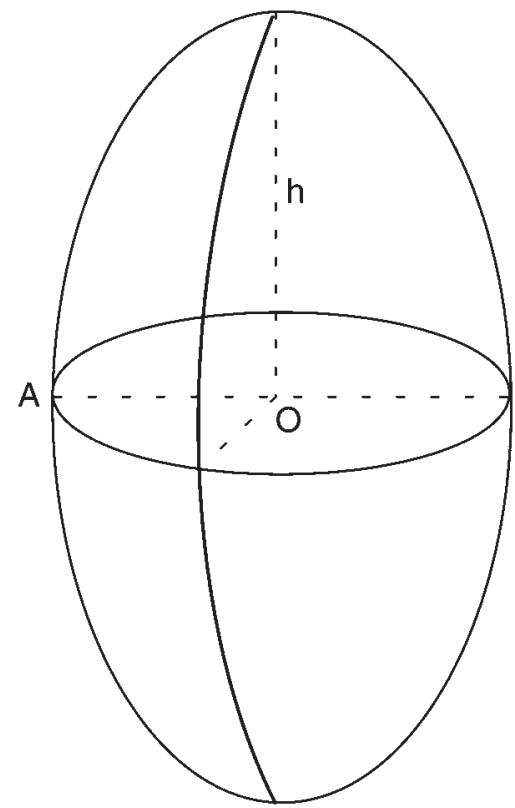

Fig. 10. An ellipsoid of revolution.

boundary $C$ of $D$, it is reflected within the disk by what is known in geometrical optics as the "law of reflection." That is, the line is reflected off the tangent to $C$ at the point the line meets $C$, in such a way that the angle of incidence of that line to that tangent is the same as the angle of reflection of that line to that tangent. At such points the geodesic of which this line is a projection passes to the second side of the "flat" ellipsoid.

The particular case of this reflection that concerns us here arises from the following configuration. Let $A$ be a diameter of the disk $D$. Let $K$ and $L$ be two lines parallel to $A$ but on opposite sides of $A$, intersecting $D$, and situated close to $A$ and at different distances from $A$. (Figure 11 depicts such a configuration.) Build two triangles inside $D$ such that the triangles are perspective from a point $O$, with $O$ inside $D$ as well; such that two of the three points of intersection of opposite sides are situated on $A$; and such that the remaining two sides are situated on $K$ and $L$, respectively. (Figure 12 depicts such a configuration, with the lines of the two triangles darker than the other lines.)

If the consequent of Desargues' theorem were to hold of these triangles, then the third point of intersection of opposite sides would also be situated on $A$. But $A$ is reflected on itself, while $K$ and $L$ are at different distances from $A$, so the intersection of $K$ and $L$ cannot be situated on $A$ (as Figure 11 illustrates in one case). ${ }^{26}$ Thus in general Desargues' theorem does not hold on ellipsoids, since it does not hold in this limiting case. ${ }^{27}$

26 Note that the reflections on $C$ of all the lines parallel to $A$ together envelope what is known in geometrical optics as a "caustic," in particular a curve known as a "nephroid," familiar as the shape yielded by light rays refracted when passing through a drinking glass; cf. Hilbert \& CohnVossen (1952, p. 280) and Audin (2003, p. 263).

27

A full proof that Desargues' theorem fails in this limiting case would use analysis, and we do not give this proof here. Doing so would be routine and in fact would have been routine even in Peano's time. 


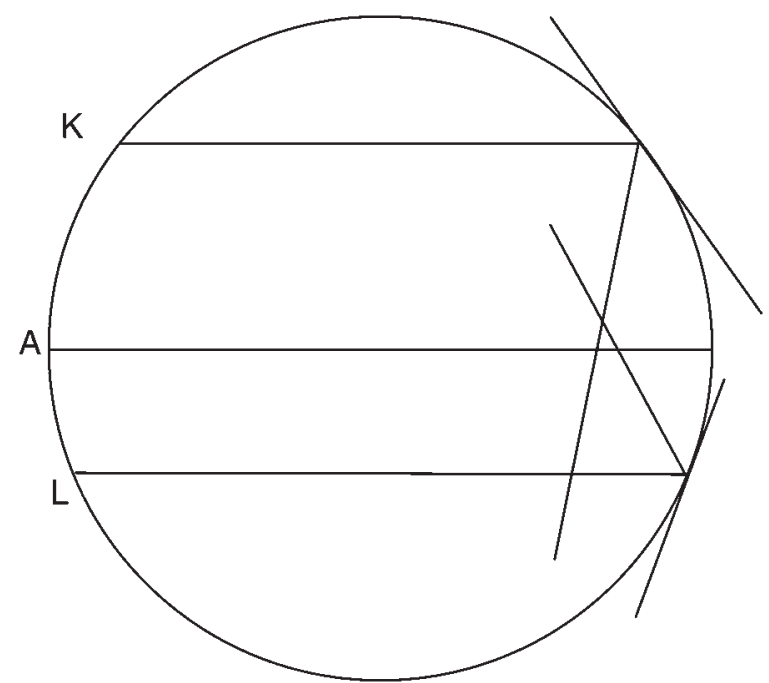

Fig. 11. A projection of the limiting ellipsoid.

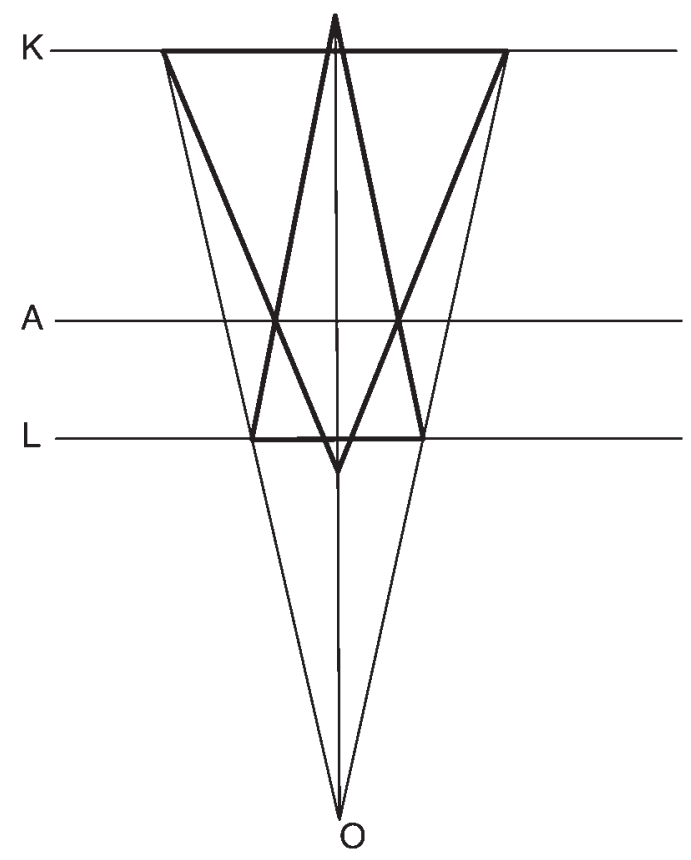

Fig. 12. A Desargues configuration for the limiting ellipsoid.

Our claim, again, is not that this construction can be found in Peano's writings. We claim only that such a construction might have been what Peano had in mind in the passage quoted above. In any case Peano's production of a non-Desarguesian plane was only implicit, since he did not provide a construction like the one we have just given. In the next section we will turn to Hilbert's more widely-known, explicit construction of a 
non-Desarguesian plane. ${ }^{28}$ We will also briefly discuss the differences between Peano and Hilbert's approaches, and why Hilbert, even if he knew Peano (1894) (we do not know whether he did), might not have thought that Peano's work had settled the matter once and for all.

3.3. Hilbert. In the Grundlagen der Geometrie, Hilbert (1899) divided his axioms into five classes: incidence axioms (I); order axioms (II); the parallel axiom (III); congruence axioms (IV); and continuity axioms (V). Within the incidence axioms in I he notes that axioms I 1 and 2 concern planar geometry, while the rest of the axioms 3-7 in I concern spatial geometry. He observed that Desargues' theorem is provable in this system using spatial axioms, or alternately using axioms of congruence. He then showed (Theorem 33) that Desargues' theorem cannot be proved in plane geometry (in fact, from axioms I 1-2, II, III, IV 1-5, and V), by presenting explicitly a model in which these axioms are satisfied but Desargues' theorem is not. ${ }^{29}$ We now narrate that presentation. ${ }^{30}$

Start with the ordinary Euclidean plane, with a coordinatization relative to the usual $x$ and $y$ axes. Construct an ellipse $\mathcal{E}$ centered at the origin of this plane, with semimajor axis 1 and semiminor axis $\frac{1}{2}$. Hilbert's model $\mathcal{H}$ of the plane is obtained from this configuration by interpreting "point" and "line" as follows. The points in $\mathcal{H}$ are just the points of the ordinary Euclidean plane. The lines that do not intersect $\mathcal{E}$ in the ordinary plane are, in $\mathcal{H}$, the same as in the ordinary plane. But in $\mathcal{H}$ lines that intersect $\mathcal{E}$ in the ordinary plane are "bent" inside $\mathcal{E}$ as follows. Say a line $\ell$ intersects $\mathcal{E}$ in the ordinary plane at points $\left(x_{0}, y_{0}\right)$ and $\left(x_{1}, y_{1}\right)$. Outside of $\mathcal{E}$, the corresponding line to $\ell$ in $\mathcal{H}$ is the same as $\ell$. But inside $\mathcal{E}$, between $\left(x_{0}, y_{0}\right)$ and $\left(x_{1}, y_{1}\right)$, the corresponding line in $\mathcal{H}$ takes the path of the arc of the circle passing through the three points $\left(x_{0}, y_{0}\right),\left(x_{1}, y_{1}\right)$, and $\left(\frac{3}{2}, 0\right)$. The diagrams in Figure 13 illustrate $\mathcal{H}$ : the top left diagram shows $\mathcal{E}$ and three Euclidean lines that intersect at $\left(0, \frac{1}{2}\right)$ in the Euclidean plane; the top right diagram shows the three circles passing through $\left(\frac{3}{2}, 0\right)$ and, for each line, the two points of intersection of that line and $\mathcal{E}$; the bottom left diagram shows the "bent" interpretations of those lines in $\mathcal{H}$; and the bottom right diagram shows these lines with higher magnification, making clearer how they do not intersect in a common point in $\mathcal{H} .^{31}$

Hilbert noted that $\mathcal{H}$ satisfies his axioms of plane geometry, and showed it does not satisfy Desargues' theorem. To see this, suppose that $\triangle A B C$ and $\triangle A^{\prime} B^{\prime} C^{\prime}$ are two triangles meeting the conditions of Desargues' theorem. Then in the ordinary Euclidean plane, the intersections $C^{\prime \prime}, A^{\prime \prime}$, and $B^{\prime \prime}$ of the corresponding sides of $\triangle A B C$ and $\triangle A^{\prime} B^{\prime} C^{\prime}$ are

28 Of course what the Peano independence result provides is a model of his axioms 1-14 in which Desargues' theorem does not hold. Which axioms of Hilbert's geometry are verified/falsified in this model would require further investigation. In other words, one would have to study the relation of the axiomatic system of geometry given by Peano and that given by Hilbert.

29 The model we will present is given in the first six editions of the Grundlagen der Geometrie. In the seventh edition onward he gives Moulton's model (cf. Moulton, 1902), which is simpler in that it does not depend on a construction involving an ellipse and circles like Hilbert's, but only on lines. He had already demonstrated the unprovability of Desargues' theorem in plane geometry in lectures in 1898/1899, though with a quite different model (cf. Hallett \& Majer, 2004, pp. 234-241; Hallett, 2008, pp. 224-225).

30 For further discussion of Hilbert's identification of a non-Desarguesian plane, and its refinement in Moulton and Moufang's work, see Cerroni (2004).

31 We have verified computationally that these lines do not intersect in a common point in $\mathcal{H}$. The computations are complicated and required the use of Mathematica. 

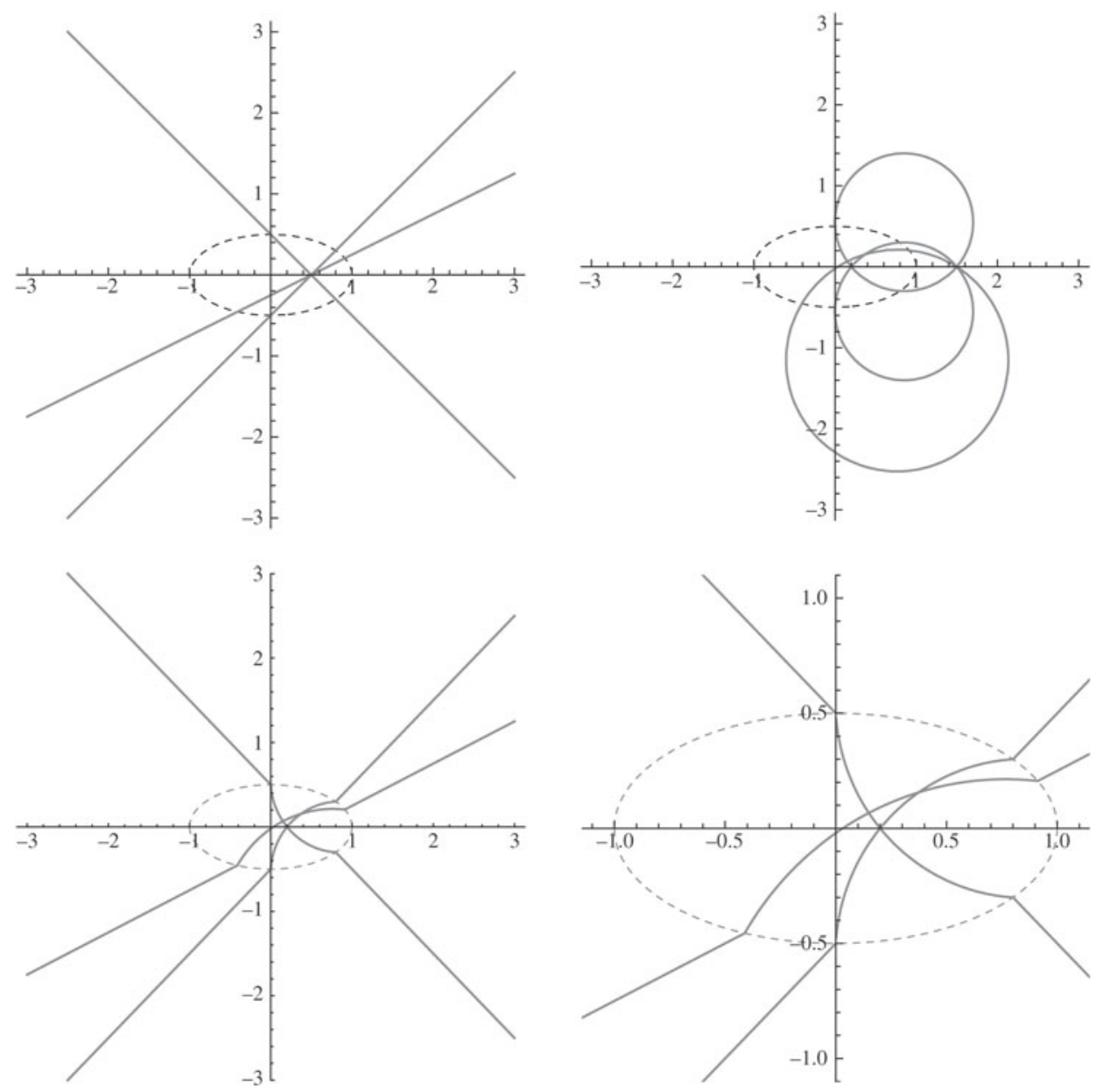

Fig. 13. Lines in Hilbert's model.

collinear, as per the conclusion of Desargues' theorem. But in Hilbert's plane $\mathcal{H}, \triangle A B C$ and $\triangle A^{\prime} B^{\prime} C^{\prime}$ may be such that $C^{\prime \prime}$ does not lie on the line $\ell$ determined by $A^{\prime \prime}$, and $B^{\prime \prime}$, because the intersection of $A B$ and $A^{\prime} B^{\prime}$ may occur inside the ellipse $\mathcal{E}$ and hence occur in a different place than it did in the ordinary Euclidean plane. We can illustrate this by reference to Figures 6 and 13: suppose that the lines $B A C^{\prime \prime}, B^{\prime} A^{\prime} C^{\prime \prime}$, and $A^{\prime \prime} B^{\prime \prime} C^{\prime \prime}$ intersect (at $C^{\prime \prime}$ ) inside $\mathcal{E}$, as in the top left diagram in Figure 13. Then in $\mathcal{H}$ they may no longer intersect, as in the bottom two diagrams in Figure 13, and thus the consequent of Desargues' theorem would not hold.

Hilbert continued by considering the "embeddability" of planes satisfying Desargues' theorem into space. In Theorem 35 in the Grundlagen (and also in the contemporaneous lectures), Hilbert shows that if a planar geometry satisfies axioms I 1-2 (the planar incidence axioms), II (the order axioms), and (in the Grundlagen) III (the parallel axiom), then Desargues' theorem is necessary and sufficient for that planar geometry to be an element of a spatial geometry satisfying all the incidence axioms I in addition to the axioms of II and III. That is, a plane satisfying axioms I 1-2, II, and III, and also satisfying Desargues' theorem, will also satisfy the spatial incidence axioms I 3-7. 
Hilbert proved Theorem 35 by showing firstly how, in a planar geometry satisfying axioms I 1-2, II, III, and Desargues's theorem, to construct an algebra of segments that is an ordered division ring (or ordered "skew field" as it is called in studies of projective geometry); and secondly how this ordered division ring can be used to construct a model in which all the axioms of I, II, and III are satisfied, that is, a model of spatial geometry. This division ring enables a coordinatization of the spatial model, and Hilbert observed that by setting the third coordinate equal to zero we have a coordinatization of the same plane with which we began. Thus one can say that a plane satisfying Axioms I 1-2, II, and III, plus Desargues' theorem, can be "embedded" into space as axiomatized by the full collection of Axioms I-III. Axioms I 1-2, II, and III without Desargues' theorem, however, do not together enable this embedding into space, since (by Theorem 33) there are models of I 1-2, II, and III in which Desargues' theorem is not satisfied, yet there are no models of axioms I, II, and III in which Desargues' theorem is not satisfied. In this sense Desargues' theorem is a necessary and sufficient condition for a plane geometry satisfying the planar incidence, order, and parallel axioms to be "embeddable" in a spatial geometry. As Hilbert put it:

Then the Desargues Theorem would be the very condition which guarantees that the plane itself is distinguished in space, and we could say that everything which is provable in space is already provable in the plane from Desargues. (Hallett \& Majer, 2004, p. 240; translation from Hallett, 2008, p. 223)

In his book with Cohn-Vossen, Hilbert described those findings as follows:

Desargues' theorem can be proved in space by using only the axioms of incidence. But in order to prove the two-dimensional form of the theorem without three-dimensional constructions, even the axioms of incidence combined with the Archimedean axiom and the axioms of order will not suffice. On the other hand, the axioms of incidence in the plane together with the axioms of order and the axioms of congruence will do, and we can dispense with the Archimedean axiom. (cf. Hilbert \& Cohn-Vossen, 1952, p. 131)

Desargues' theorem seems to concern just the incidence and intersection of lines and points, and as Hilbert acknowledged, can be proved using just incidence axioms, albeit in spatial versions (for such a proof, cf. Hartshorne, 1967, theorem 2.1). If we try to avoid these spatial axioms, we cannot prove Desargues' theorem even by adding order or parallel axioms, since the model of a non-Desarguesian plane discussed in Section 3.3 satisfies Hilbert's order and parallel axioms as well. As a result, "its demonstration necessitates either the space axioms or all of the axioms of congruence," as Hilbert put it in Theorem 33 in the Grundlagen.

Having now documented Hilbert's geometrical accomplishment-documentation that will be essential in the remainder of the paper-we turn to a comparison of Hilbert's accomplishment with Peano's.

3.4. Comparing Peano and Hilbert's results. In our view, Peano deserves priority for having first found a non-Desarguesian plane. However, as we saw earlier, Peano did not explicitly specify his non-Desarguesian plane. Instead, he relied on an implicit understanding of the differential geometry of surfaces, using this to suggest how one could find 
non-Desarguesian planes by considering the geometry of geodesics on surfaces of nonconstant curvature. While we gave a construction that plausibly could have been one that he had in mind, the fact remains that Peano's failure to provide an explicit specification meant that geometers could have reasonably desired more. We do not know whether Hilbert knew of Peano (1894), but even if he did, it is plausible that he desired a more explicit specification of a non-Desarguesian plane. We have thus found an explanation of why Hilbert saw fit to reprove Peano's result, even supposing that he knew of it.

On the other hand, Hilbert's model is rather artificial, in that it was constructed simply for the purpose of falsifying Desargues' theorem. Peano's idea, by contrast, arises naturally from the study of elementary surfaces in differential geometry. Hilbert's model signifies an advance of another kind, though. While Peano's work adhered to the nineteenth century limitation to surfaces endowed with smooth metrics, as discussed in Section 3.1, Hilbert's model represents a surface that fails to be smooth on the boundary of the ellipse $\mathcal{E}$, and hence can be seen to be a non-Riemannian surface. At the time of his investigations into Desargues' theorem Hilbert was also promoting a widening of the scope of investigation of metrical geometries, for instance in the fourth problem of his 1900 address in Paris to the International Congress of Mathematicians. His specification of a non-Riemannian, non-Desarguesian plane thus fits into this wider research program. In Section 4.6 we will consider in further detail the significance of this research program for the case of Desargues' theorem.

§4. Purity and Desargues' theorem. Having brought into focus Desargues' theorem in its historico-mathematical and foundational context, we now return to the philosophical problems raised but left unanalyzed in our discussion of the fusionist debate in Section $\S 2$. What is it for a solution to a problem, or a proof of a theorem, to draw on considerations "foreign" to its content? What is the appropriate notion of "content" that can support an adequate account of talk of purity in mathematical practice? How can this notion of content be articulated analytically? In this section we will address these questions by zeroing in on them in regard to Desargues' theorem as set out in the previous section.

We will proceed as follows. First we begin in Section 4.1 with Hilbert's pronouncements on the content of Desargues' planar theorem and how this relates to purity. We point out that Hilbert seems to judge such a pure proof impossible, since he takes his work to show that solid considerations are necessary for proving Desargues' theorem in his projective axiomatic setting, but holds that solid considerations do not belong to the "content" of Desargues' theorem and for this reason an appeal to such considerations cannot yield a pure proof of the theorem. We will then in Section 4.2 give a brief account of Hilbert's axiomatic method. This account will be instrumental in resisting a radical "formalist" interpretation of Hilbert's conception of the axiomatic method and thus to give proper weight to Hilbert's claims about the "content" of Desargues' theorem against a possible reading that, resting on a radical formalist reading of the axiomatic method, would relegate them to mere loose talk. In Section 4.3 we look at how one of the most influential commentators of Hilbert's work in geometry, Michael Hallett, conceives of the relationship between content and purity in Hilbert's work. Despite some hesitation, Hallett favors a construal of the content of Desargues' theorem that has as a consequence that the proof of Desargues' planar theorem by means of solid considerations is pure. According to Hallett, solid proofs of Desargues' theorem are not necessarily impure, since, it is argued, Desargues' theorem has "tacit spatial content." After getting clearer in Section 4.4 on exactly what notion of "content" is salient for Hallett's 
interpretation, we argue in Section 4.5 against Hallett concerning the purity of the classical solid proofs of Desargues' theorem, on the basis of problems we raise for Hallett's notion of content, which we claim is inappropriate in evaluations of purity. Finally, in Section 4.6, we discuss another way to think about the content of Desargues' theorem, namely, as a theorem with metrical content, contra the usual understanding of the theorem as projective.

One final caveat before we proceed. We do not deny that a notion of "formal" or "deep" content, as propounded by Hallett, might be of interest. We do however claim, and present several arguments to this effect, that this is not the notion of content that can be serviceable for an account of purity. The notion of content that we deem necessary in clarifying mathematicians' judgments about purity is one that is tied to the ordinary understanding of mathematical statements.

4.1. Hilbert on purity of methods. Hilbert's interest in purity at the end of the $1890 \mathrm{~s}$ is well established. ${ }^{32}$ In his 1900 Paris address on mathematical problems, Hilbert articulated a purity constraint as desirable in the solution of such problems:

It remains to discuss briefly what general requirements may be justly laid down for the solution of a mathematical problem. I should say first of all, this: that it shall be possible to establish the correctness of the solution by means of a finite number of steps based upon a finite number of hypotheses which lie in the presentation of the problem and which must always be exactly formulated. (Cf. Hilbert, 1901, p. 257)

He emphasized his interest in purity in particular in describing his investigation of the result we have been discussing, Desargues' theorem. As he put it in lectures in 1898/1899:

This theorem gives us an opportunity now to discuss an important issue. The content [Inhalt] of Desargues' theorem belongs completely to planar geometry; for its proof we needed to use space. Therefore we are for the first time in a position to put into practice a critique of means of proof. In modern mathematics such criticism is raised very often, where the aim is to preserve the purity of method [die Reinheit der Methode], i.e. to prove theorems if possible using means that are suggested by [nahe gelegt] the content of the theorem. (cf. Hallett \& Majer, 2004, pp. 315-316)

Thus Hilbert judged a proof to be pure just in case it draws upon only what is "suggested by the content of the theorem" being proved, or on what "lies in" the presentation of

32 It is important to note this concentration because while one may reasonably view Hilbert's finitist project as aspiring toward a type of purity (cf. Kreisel, 1980 for such a suggestion), it is not clear that it aspired to the same type of purity that interested him at the turn of the century. If the finitist project is to be understood as representing a purity constraint on proof at all, it is a constraint that limits the resources available to proofs to those of a certain minimal or "basic" epistemic status, rather than to those "contained in" the content of the theorem being proved. The link, if any, between these types of purity constraint deserves further investigation; but it should be clear that these constraints are not necessarily the same. For a general historical survey of purity see Detlefsen (2008), and for an analysis of the epistemic value of the type of purity with which Hilbert seems to have been concerned, see Detlefsen \& Arana (2011). 
the problem being solved. ${ }^{33}$ Since he judged that the "content of Desargues' theorem belongs completely to planar geometry," solid considerations seem not to be "suggested by the content of the theorem," and therefore it would seem that he judged solid proofs of Desargues' theorem to be impure. ${ }^{34}$

Hilbert sought further to answer whether there could be a pure proof of Desargues' theorem, expressing his goal as follows:

I have said that the content [Inhalte] of Desargues's Theorem is important. For now however what's important is its proof, since we want to connect to this a very important consideration, or rather line of enquiry. The theorem is one of plane geometry; the proof nevertheless makes use of space. The question arises whether there is a proof which uses just the linear and planar axioms.... (cf. Hallett \& Majer, 2004, p. 236; translation from Hallett, 2008, p. 223)

In this passage he stresses again that the content of Desargues' theorem is purely planar, and asks whether there could be a proof of it that avoids solid considerations in favor of purely planar considerations. Hilbert's Theorem 33 suggests that this is possible if the proof appeals to metric considerations such as axioms of congruence, but not otherwise. Supposing that metric considerations are not appropriate for a pure proof of Desargues' theorem (a supposition to which we will return in Section 4.6), Hilbert's result seems to show that no pure proof of Desargues' theorem is possible.

Such, at any rate, seems to have been Hilbert's take on this. His take deserves further scrutiny, though, on account of its rather casual invocation of the "content" of a theorem and of what is "suggested" by or what "lies in" it. We thus turn to a more careful discussion of these notions. We want to draw attention in particular to two notions of content that might be thought relevant to purity. The first we will call the "informal" or "intuitive"

33 Hilbert also stressed the implications of his work for purity considerations in the Grundlagen. As he wrote in its epilogue,

In this investigation the ground rule was to discuss every question that arises in such a way so as to find out at the same time whether it can be answered in a specified way with certain limited means... The ground rule according to which the principles of the possibility of a proof should be discussed at all is very intimately connected with the requirement for the "purity" of the methods of proof which has been championed by many mathematicians with great emphasis. This requirement is basically none other than a subjective form of the ground rule followed here. (Cf. Hilbert, 1971, pp. 106-107)

He stressed that the "subjectivity" to which he referred was the mathematician's prerogative to determine for herself what means of proof she preferred.

34 In a recent article (Webb, 2006) Judson Webb describes a distinction drawn by Peirce between proofs that are "corollarial" and "theorematic," which is in the vicinity of our distinction between pure and impure proofs, respectively. A proof of a theorem is "theorematic" if it draws on "more than what the condition supposes to exist" (quoted in Webb, 2006, p. 249). Peirce observed that the classical metrical proof of Desargues' theorem is "theorematic" because it "introduced an idea [of length] which was in nowise involved in the premises," and that the classical solid proof is "a very good example" of theorematic reasoning. Webb observes that Peirce thought one should try to give corollarial proofs when possible, but, having learned of Hilbert's metageometrical work in the Grundlagen, concluded that no such proof was possible for Desargues' theorem (cf. Webb, 2006, p. 251; also cf. Webb, 1980, pp. 93-97). For the purposes of this paper we do not need to investigate further the distinction between corollarial and theorematic proofs in Peirce. 
content of a statement, by which we mean what someone with a casual understanding of geometry would (be able to) grasp. The second we will call the "formal" or "axiomatic" content of a statement, by which we mean the inferential role of that statement within an axiomatic system (referees suggested "propositional content" or "logical content" for this notion). ${ }^{35}$ On a first glance these are different notions of content, since the latter involves inferential connections that seem to have nothing to do with informal or intuitive understanding. The question then becomes: which notion of content yields a purity constraint that is truer to practice, including Hilbert's practice? Our contention is that it is the first, informal notion of content. To make our case, we will consider what we take to be a radical reading of Hilbert in which the "content" at stake in purity considerations is formal, as exemplified by a recent article Hallett (2008) by Michael Hallett. We will show several reasons why this reading is problematic for understanding both Hilbert's views on purity and purity in general, and how reading content as informal alleviates those problems. This is not to deny that the notion of formal content is an important and valuable one to single out for analysis; only that it does not seem to us to be true to ascriptions of purity in mathematical practice. ${ }^{36}$ And if, contrary to what we claim, Hilbert had effected a transformation of traditional purity concerns with concomitant elimination of the notion of "intuitive" content in favor of a new "formal" notion of content, then Hallett's reading of Hilbert would be right but we would then have to raise the same criticisms of descriptive inadequacy — vis-à-vis ascriptions of purity in mathematical practice- to Hilbert too. But first we need to say something about Hilbert's axiomatic method.

4.2. Hilbert's axiomatic achievement. Hilbert's conception of the axiomatic method has been described many times. One of the key issues in interpreting what the method amounts to and differs from other axiomatic approaches, such as that of Euclid in the Elements, concerns the relation between intuition and formalism. This turns out to be the central issue for us and we readily grant that our reading of Hilbert is not the only one plausible; however, it is the reading that makes best sense to us. Briefly, we will claim that a radical interpretation of Hilbert's conception of axiomatic geometry leaves room only for a notion of content that is useless for judging issues of purity as understood in mathematical practice. By contrast, our interpretation does not put such a cleavage between intuition and formalism and has the advantage, in our opinion, of being more historically accurate and of accounting for Hilbert's claim that the content of Desargues' planar theorem is planar without dismissing Hilbert's claims on the matter as mere loose talk.

Hilbert's most famous contribution to the foundations of mathematics is the Foundations of Geometry, published in 1899. The goal of the work is to investigate the field of geometry axiomatically. This led Hilbert to substantial revisions of the approach taken in the Euclidian Elements. The most fundamental distinction between Euclid's approach to geometry and Hilbert's, the old and the new axiomatics, consists, to speak with Bernays, in a "new methodological turn" in the conception of axiomatics. When we open Euclid's Elements, we are presented with axioms and notions that are already interpreted; that is, when using the word "point" or "line," Euclid is referring to a specific object of geometrical intuition.

35 While "formal" has many associations that might lead one to recommend one of the other suggested modifiers instead, each modifier has its own advantages and disadvantages, and on the whole we prefer "formal" to the alternatives.

36 For a survey of such ascriptions from mathematical practice, see Detlefsen (2008a) and Detlefsen \& Arana (2011). 
The axioms are true of these objects. The axioms thus organize the body of geometrical knowledge by showing from which true propositions the rest of the geometrical truths can be derived by means of logical inference. Hilbert invites us to reach a higher level of generality. Rather than anchoring from the start the basic concepts (point, line, plane) to our pregiven geometrical understanding of those notions, he asks us to consider any three systems of objects and three sorts of relations between these objects (lie, between, congruent). The axioms of geometry then only state how these relationships relate the objects in question. As Bernays remarks, this led to the separation between the mathematical and the epistemological problems of axiomatics.

The important thing, then, about Hilbert's Foundations of Geometry was that here, from the beginning and for the first time, in the laying down of the axiom system, the separation of the mathematical and logical [spheres] from the spatial-intuitive [sphere], and with it from the epistemological foundation of geometry, was completely carried out and expressed with complete clarity. (Cf. Bernays, 1922, p. 192)

In Hilbert's approach the axioms give an implicit definition of the relevant concepts. Let us quote Bernays again:

According to this conception, the axioms are in no way judgments that can be said to be true or false; they have a sense only in the context of the whole axiom system. And even the axiom system as a whole does not constitute the statement of a truth; rather, the logical structure of axiomatic geometry in Hilbert's sense, analogously to that of abstract group theory, is a purely hypothetical one. If there are anywhere in reality three systems of objects, as well as determinate relations between these objects, such that the axioms of geometry hold of them (this means that by an appropriate assignment of names to the objects and relations, the axioms turn into true statements), then all theorems of geometry hold of these objects and relationships as well. Thus the axiom system itself does not express something factual; rather, it presents only a possible form of a system of connections that must be investigated mathematically according to its internal properties. (Bernays, 1922a, p. 192)

So far what we have said can be interpreted as disconnecting completely intuition and formalism thereby rendering any talk of "informal content" (as opposed to "formal content" or "logical weight of a proposition") of a formalized geometrical statement useless. Indeed, if we are dealing only with a framework of uninterpreted symbols there is not even much sense in saying that the axioms are geometrical axioms; after all, they could be just as well interpreted as being about chairs, tables and beer mugs! No privileged notion of content is available to say that a statement in the system is a statement of geometry as opposed to any other domain. But we claim that for Hilbert the formalism is only methodological. In other words, while it is useful for certain methodological aims to treat the formalism as having no meaning (and therefore subject it to radical reinterpretation) this does not mean that the intimate connection between the intuitive truths of geometry and the formalism is abandoned. Thus, one should not rashly conclude that for Hilbert intuition no longer plays any role. Intuition is essential for suggesting which axioms we need to select in order to account for our geometrical knowledge, and having done that, we need to take an extra step by going beyond intuition to the consideration of any three systems of objects that might act as reference of the words "point," "line," and "plane." 
However, and this is the key point, a certain parallelism is maintained between the intuitive and the formal axiomatic level.

It is worthwhile to emphasize this point for two reasons. Firstly, Hilbert's view of mathematics has often been reproached for reducing mathematics to a mere study of formalisms, a pure game with symbols. However, it is enough to peruse the 1919 lectures (cf. Hilbert, 1992) to realize how far this is from Hilbert's view of the matter. Secondly, Hilbert's stress on the parallelism between the intuitive content and the formalism is central to our claim that it is perfectly consistent for Hilbert to defend methodologically a radical formalism while holding on to the notion of content corresponding to the privileged interpretation of the system. Consider for instance what Hilbert says in a series of lectures given in Göttingen in 1905. Hilbert explains, in analogy with his work in geometry, the new abstract approach to the axiomatic treatment of numbers as follows:

The numbers have become for us only a framework of concepts to which we are led of course only by means of intuition; we can nonetheless operate with this framework without having recourse to intuition. However, to insure the applicability to the objects surrounding us, this conceptual system is constructed in such a way that it forms everywhere a complete analogy with the most trivial intuitions and with it to the facts of experience. (quoted in Peckhaus, 1990, p. 60) (37 $^{37}$

The parallelism emphasized by Hilbert is our reason for holding that it is perfectly consistent for Hilbert to assign a planar content to the statement of Desargues' planar theorem. ${ }^{38}$ Notice that our position does not exclude an alternative notion of "formal"

37 Peckhaus' quote from Hilbert (1905a) reads, "Die Zahlen sind für uns nur ein Fachwerk von Begriffen geworden, auf das wir allerdings nur durch di Anschauung geführt werden, mit dem wir aber desungeachtet ohne jede Zuhilfenahme der Anschauung operieren können. Damit aber dies Begriffssystem auf die uns umgebenden Dinge anwendbar wird, ist es von Grund aus so construiert worden, dass es überall eine volle Analogie mit den trivialsten Anschauungen und damit den Tatsachen der Erfahrung bildet." See also Hilbert (1905b, p. 36): “Cap. II Axiome der Geometrie. Wir kommen nun zu dem Aufbau der Geometrie, in der die Axiomatik am ersten vollständig durchgeführt wurde. Wir sind beim Aufbau der Arithmetik von deren anschauliche Grundlage, dem Anzahlbegriff, schliesslich ganz abgekommen, den ja nach bei der genetischen Methode den Ausgangspunkt bildet. Uns war das Zahlensystem wirklich nichts, als ein Fachwerk von Begriffen, das durch 18 Axiomen definiert war. Bei der Aufstellung dieser bietete uns allerdings der Anschauung, die wir von dem Begriff der Anzahl und seiner genetischen Ausdehnung haben, denn das Ziel ist und bleibt natürlich ein möglichst leicht und klar anwendungs-fähiges Zahlensystem zu haben; dies Ziel wird ersichtlich die eine klare Axiomatik besser erreicht als durch jede andere Definitionen. So ist in jeder Wissenschaft die Aufgabe in den Axiomen zunächst ein Fachwerk von Begriffen zu errichten, bei dessen Aufstellung wir uns natürlich durch die Anschauung und Erfahrung leiten lassen; das Ideal dann, dass in diesem Fachwerk alle Erscheinungen des letzten Gebietes Platz finden, und dass jedem aus den Axiomen folgende Satz dabei Verwertung findet. Wollen wir nun für die Geometrie ein Axiomsystem aufstellen, so heisst das, dass wir uns den Anlass dazu durch die anschaulichen Thatsachen der Geometrie [...] lassen und diesen das aufzurichtende Fachwerk entsprechen lassen; die Begriffe, die wir so erhalten, sind aber als gänzlich losgelöst von jedem Erfahrung und Anschauung zu betrachten."

38 Let us say a bit more about this parallelism. When working axiomatically we are forced to choose a type of axiomatization: for instance, in axiomatizing the concept of straight line, we must choose between affine, projective, and metrical axiomatizations (as we will discuss more closely in Section 4.6). When working informally, though, we must make a parallel choice, concerning how the concept of straight line is (to be) understood. These choices can inform each other. As 
content (see below our discussion of Hallett on "hidden higher-order" content). We just deny that the latter is the only notion of content available to Hilbert. In this way, we can accept Hilbert's statement about the planar content of Desargues' planar theorem without having to accept that such talk loses meaning once one comes to appreciate the radical departure from intuitive content required by Hilbert's axiomatic method.

4.3. Hallett on purity in Hilbert. We turn now to Hallett's reading of Hilbert in Hallett (2008), as an exemplar of the "formal" construal of content in understanding Hilbert's purity constraint. Hallett observes firstly that Desargues' theorem "appears to involve only planar concepts, i.e., is concerned only with the intersection of lines in the same plane" (p. 223). As a result, he comments, a spatial proof of Desargues' theorem "at first sight might be thought inappropriate, owing to the proof's appeal to spatial axioms" (p. 224); that is to say, "at first sight" a solid proof of Desargues' theorem appears impure.

We may read Hallett as identifying this "first sight" representation of the theorem's content as what we are calling its informal content. The locution "at first sight" signals, though, that Hallett believes further investigation casts doubt on the inappropriateness or impurity of spatial axioms in proving Desargues' theorem. Hallett makes such a case by taking the content of Desargues' theorem to be what we are calling its formal content. We will next narrate the details of his case.

Hallett begins by observing that while a solid proof of Desargues' theorem may appear impure "at first sight," Theorem 35 indicates otherwise. He describes Theorem 35 as showing that "the original plane (now characterized by coordinate pairs) will be a plane in the new space; moreover, the fact that the right incidence axioms hold for planes means that this plane will be "properly' embedded in the space" (pp. 228-229). This has the following consequence, Hallett claims:

What this shows is that that [sic] the Planar Desargues's Theorem is a sufficient condition for the orderly incidence of lines and planes, in the sense that it can be used to generate a space. We thus have an explanation for why the Planar Desargues's Theorem cannot be proved from planar axioms alone: the Planar Desargues's Theorem appears to have spatial content. (p. 229)

That is, while Hilbert held that 'the content of Desargues' theorem belongs completely to planar geometry," Hallett claims that Desargues' theorem has spatial content that is revealed by Hilbert's proof of Theorem $35 .{ }^{39}$ He explains the significance of this claim as follows:

[W]hat [Hilbert's work] reveals is that the Planar Desargues's Theorem in effect actually has spatial content. This provides an explanation of why it is (in the absence of congruence and the Parallel Axiom) that the Planar Desargues's Theorem cannot be proved without the use of

we read Hilbert, his formal investigations were aimed at shedding light on the geometry of "the objects surrounding us," and to the geometry given to us by Euclid. But it should not be thought that we can escape these choices by settling on a particular construal of the informal content of a statement, for it is just this settling on a particular construal that can be clarified by the kind of formal work Hilbert carries out.

39 Incidentally, it is not clear why one should not reverse the direction of implication and, insisting on the intuition that Desargues' theorem has planar content, conclude that the solid axioms have planar content. 
spatial assumptions, and it provides a beautiful example of Hilbert establishing in the fullest way possible why 'impure' elements are required in the proof of Desargues's Theorem, the grounds for the 'impossibility of success' in trying to prove Desargues's Theorem in the plane. (pp. 227-228)

Although in the above quote Hallett seems to claim that Hilbert's result shows that a proof of the planar Desargues theorem must be impure, his considered position turns out to be different. Indeed, he suggests that since the content of the planar Desargues theorem is spatial rather than planar, solid projective proofs of Desargues' theorem in fact draw only on what is "suggested by the content of the theorem," and hence are pure.

Hallett observes that this judgment rests on abandoning an informal construal of the theorem's content. As he puts it:

[T] he cases examined are all ones where the appropriateness of an assumption is initially questioned, but where it is shown that it is indeed required. Among other things, this forces a revision of what is taken to be 'appropriate'. It is part of the lesson taught by the examples that in general this cannot be left to intuitive or informal assessment, for instance, the intuitive assessment of the complexity of the concepts used in the assumption in question. (p. 248)

Hallett thus rejects the informal construal of the content of theorems in assessing purity claims, maintaining that results like Hilbert's can justify revising our judgments of what is "appropriate" or pure in proving theorems, even if those revisions run counter to our "intuitive or informal assessment." He is even more explicit about this in his introduction to Hilbert's 1898/1899 lectures, writing that Hilbert's work "reveals that Desargues' planar Theorem has hidden spatial content, perhaps showing that the spatial proof of the Planar Theorem does not violate 'Reinheit' after all” (cf. Hallett \& Majer, 2004, p. 197).

Hallett's view is thus that Hilbert's Theorems 33 and 35 together show that Desargues' theorem has solid content, and thus that solid considerations can be used in a pure proof of the result. Metageometrical work like Hilbert's can show that informal, "at first sight" construal's of a statement's content are wrong. We will now turn to a more focused analysis of what Hallett thinks is the "proper" way to construe said content, which we called earlier a "formal" or "axiomatic" construal of content.

4.4. Formal content. While the key to Hallett's judgment that the classical solid projective proofs of Desargues' theorem are pure is his view that Desargues' theorem has solid content, precisely what he means by "content" needs further treatment. Since an ordinary informal understanding of Desargues' theorem indicates that its content is purely planar, as Hilbert observed, Hallett recognizes that his construal of its content is not this ordinary one. He describes his construal as follows:

The examples we have considered show that often we have to adopt a non-elementary point of view in order to achieve results about apparently elementary theorems.... [T] hey also show that apparently elementary propositions contain within themselves non-elementary consequences, often in a coded form. (p. 249)

Hallett does not explain further what he means by "coded" content, offering instead a footnote to this last sentence in which he notes that "[t]here is surely here more than 
an analogy with the 'hidden higher-order content' stressed by Isaacson in connection with the Gödel incompleteness phenomena for arithmetic”, citing Daniel Isaacson's paper (Isaacson, 1996) for reference. Thus we will turn to Isaacson's work to understand Hallett's theory of content. ${ }^{40}$

Isaacson's (1996) central claim is that sentences in the first-order language $\mathcal{L}_{\mathrm{PA}}$ of arithmetic may have purely arithmetical content, or may have in addition "higher-order", that is, infinitary, nonarithmetical content. Since the ordinary understanding of sentences in $\mathcal{L}_{\mathrm{PA}}$ involves only arithmetical content, he says that their higher-order content, if any, is only "implicit" or "hidden" (hence the article's title).

That sentences in $\mathcal{L}_{\mathrm{PA}}$, particularly those true of the natural numbers, can have higherorder nonarithmetical content, is a consequence of Isaacson's view that the content of arithmetical sentence is determined by what is necessary and sufficient for "perceiving" that that sentence is true. He puts it as follows:

[A] truth expressed in the (first-order) language of arithmetic is arithmetical just in case its truth is directly perceivable on the basis of our (higher-order) articulation of our grasp of the structure of the natural numbers or directly perceivable from truths in the language of arithmetic which are themselves arithmetical. (cf. Isaacson, 1996, p. 217)

In the first case, a sentence in $\mathcal{L}_{\mathrm{PA}}$ that is true of the natural numbers is arithmetical just in case we can come to "directly perceive" its truth by way of "articulating" our grasp of the structure of the natural numbers, as Isaacson claims we do with the axioms of first-order Peano Arithmetic (PA) by recognizing their relation to a higher-order characterization like Dedekind's of the structure of the natural numbers. In the second case, a sentence in $\mathcal{L}_{\mathrm{PA}}$ true of the natural numbers is arithmetical just in case we can come to "directly perceive" its truth on the basis of other arithmetical sentences in $\mathcal{L}_{\text {PA }}$. That is, the second case classifies a sentence in $\mathcal{L}_{\mathrm{PA}}$ as arithmetical if it can be proved in PA.

Isaacson argues that on this criterion, Gödel sentences and other such truths in $\mathcal{L}_{\mathrm{PA}}$ unprovable in PA (for instance, the Paris-Harrington sentence) have hidden higher-order content. As Isaacson puts it, this is because these sentences are

shown to be true by an argument in terms of truths concerning some higher-order notion, and in each case also a converse holds, so that the only way in which the arithmetical statement can be established is by an argument which establishes the higher-order truth. The relationship of coding constitutes a rigid link between the arithmetical and the higherorder truths, which pulls the ostensibly arithmetical truth up into the higher-order. (pp. 220-221)

Thus Gödel sentences and the like are proved (over PA) equivalent to a sentence expressing by coding a metamathematical property of PA (such as consistency) that is provably unprovable in PA but provable by higher-order means. Such equivalences reveal "something of the implicit (hidden) higher-order content" of truths in the language of arithmetic, Isaacson believes, and indeed he holds (p. 214) that "the understanding of these sentences

40 While we will end up criticizing and rejecting Hallett's notion of coded or tacit content, we are not excluding that a finer notion of tacit content than the one offered by Hallett might not in fact be helpful for sorting out some of the issues we are raising. As John MacFarlane has pointed out to us, there might be a connection here to discussions in philosophy of language, such as those involving Sellars and Brandom, on the issue of whether certain ordinary descriptive concepts implicitly involve modal notions. The topic is however too large to be investigated here. 
rests crucially on understanding this coding and our grasp of the situation being coded." In virtue of having the same inferential role as sentences expressed in higher-order terms, these sentences can be said to have higher-order content, despite being expressed in purely arithmetical terms.

It is this view of Isaacson's that Hallett has in mind when writing that "apparently elementary propositions contain within themselves non-elementary consequences, often in a coded form." Hallett's view seems to be that because Desargues' theorem has the same inferential role as the spatial incidence axioms within Hilbert's geometry, it has spatial content. Hallett is explicit that its content is spatial because of its inferential role:

Moreover, as is clear in Hilbert's statement of his conjecture and from his construction, Desargues's Theorem could now be taken as an axiom to act in place of the spatial incidence axioms. The use of the theorem as an alternative axiom is quite standard in modern treatments of projective geometry, especially in treatments of affine planes...(p. 229)

Hallett says that his view is analogous to Isaacson's view that some sentences in purely arithmetical terms have higher-order nonarithmetical content.

He does not say exactly what is analogous to the coding mechanism of PA in the case of Desargues' theorem, but the idea seems to be that it is either the algebra of segments permitted by Desargues' theorem in the presence of axioms I 1-2, II, and III, or more directly the alleged spatial content it inherits from its stereometrical proof. Regardless, in both cases, we have sentences whose ordinary understanding indicates that it has content of one type (e.g., arithmetical or planar), but that an analysis of the sentences' inferential roles reveals that these sentences have tacit content of another type (e.g., infinitary or spatial, respectively).

Let us turn our attention to the account of content at work in Isaacson and Hallett's views, giving rise to their (evidently shared) conception of tacit content. As Hallett sees it, the meanings of the primitive terms of Hilbert's geometry are not fixed by reference to a single interpretation, since they may be reinterpreted in many different ways. The question then remains how the content of sentences involving these primitive terms is determined. Hallett's answer seems to match Carnap's in Logical Syntax of Language (cf. Carnap, 1937), who writes (p. 42) that " $t$ t]he question whether two sentences have the same logical sense is concerned only with the agreement of the two sentences in all their consequence-relations." There Carnap contrasts this "logical" construal of meaning with "psychological" construals of meaning for which the "images" associated with a sentence are determinative. Hallett's view of content seems to agree with Carnap's; as Hallett puts it:

Hilbert's axiomatic method abandons the direct concern with the kind of knowledge the individual propositions represent because they are about the primitives they are, and concentrates instead on what he calls 'the logical relationships' between the propositions in a theory. (p. 212)

That is, what we are calling the "formal" content of Desargues' theorem and other theorems of axiomatic geometry is determined by their inferential role within axiomatic theories. As Hallett sees it, Hilbert's Theorems 33 and 35 together show that Desargues' theorem plays the inferential role of a spatial sentence. Hence he judges Desargues' theorem to have spatial content; or, as we would put it, spatial formal content. As a result the classical solid proofs of Desargues' theorem may be judged pure, as they draw only upon resources "suggested by" or "contained" in the (formal) content of the theorem. 
4.5. Against construing purity in terms of formal content. In Sections 4.1 and 4.2 we provided textual evidence for what we take to be the straightforward reading of Hilbert's views on purity, in which the classical solid proofs of Desargues' theorem are judged to be impure. A pure proof of a theorem is one that draws on what is "suggested by the content of the theorem," as Hilbert put it. Since Hilbert says that "the content of Desargues' theorem belongs completely to planar geometry," we hold that the impurity of the classical solid proofs of Desargues' theorem follows. Hallett disputes this in light of Hilbert's metamathematical results concerning the inferential role of Desargues' theorem within his axiomatic geometry; he maintains that Desargues' theorem has tacit spatial content, and so pure proofs of Desargues' theorem may draw on solid considerations. The crux of our disagreement with Hallett concerns which notion of content is operative in ascriptions of purity in mathematical practice. We maintain that the relevant notion of content is what we have called "informal" content, the one available to ordinary practitioners. Hallett maintains by contrast that it is what we have called "formal" content, revealed by metamathematical work. We now offer a series of reasons against construing purity in terms of formal content. ${ }^{41}$

We begin by pointing out that if one infers from the multiple instantiability of Hilbert's geometry that the geometrical primitives are meaningless, then the problem of the "content" of geometrical statements cannot be raised at all, or the notion of "content" so obtained would be so different from what we understand ordinarily by such notion as to render it useless for determinations of purity (indeed, we will claim that this latter notion would trivialize purity). Hallett's interest in the problem of content, and of the difference between planar and spatial content in particular, would be difficult to understand if he held this strict formalist stance. What would it mean to claim that a formal statement is about geometrical entities as opposed to anything else, given the radical underdetermination of the formalism? We can make sense of the way that Hallett uses and talks of content by considering instead specific intuitive interpretations of the axioms/sentences in question. In particular we can consider specific intuitive interpretations of Hilbert's axioms, following the ordinary understanding of the defined terms, so that the sentences of Hilbert's geometry have, at least in part, the content we ordinarily ascribe to them. This way of proceeding corresponds to the construal we offered in Section 4.2 of Hilbert's axiomatic approach.

41 We may settle another issue with Hallett's reading of Hilbert as follows. On p. 222 of Hallett (2008), the subject is identified as "Hilbert's treatment of Desargues' Theorem in elementary projective geometry." While Hilbert's 1891 lectures explicitly concern projective geometry, in the later 1898-1899 lectures and the Grundlagen both affine and projective geometry are investigated. Indeed, in the Grundlagen, Hilbert states Desargues' theorem in affine rather than projective terms. Hallett acknowledges this difference between the 1898-1899 lectures and the Grundlagen on p. 226, but does not note the following significance for investigations of purity: Hilbert's affine formulation justifies the purity of his appeal to the parallel axiom III in proving Desargues' theorem, while an appeal to parallels for the result stated projectively would seem to be impure. In the 1898-1899 lectures he avoids the affine formulation and finds both nonaffine and affine nonDesarguesian planes, the latter by way of arguing that adding the parallel axiom to the planar axioms of classes I and II does not enable the proof of Desargues' theorem. Since the formulation of Desargues' theorem there avoids parallels, this addition would have evidently been an impurity. Hallett presents Hilbert's nonaffine non-Desarguesian plane in his Figure 8.2 (p. 225) and the surrounding text (Hallett \& Majer, 2004, pp. 316-318), but not the affine non-Desarguesian plane presented later in those lectures (Hallett \& Majer, 2004, pp. 376-377). For detailed technical investigations of Hilbert's models in both the lectures and the Grundlagen, including both his affine and nonaffine models, cf. Schneider \& Stroppel (2007) and Stroppel (2010). 
Let us consider critically what Hallett's view of tacit content implies for the case at hand, Desargues' theorem. If the content of Desargues' theorem is spatial, as Hallett would have it, it would seem to follow that an investigator with no beliefs concerning space could not understand Desargues' theorem. Consider, for example, one of the characters in the novel Flatland (cf. Abbott, 1884), such as A. Square, who is (at least initially) familiar with nothing outside the plane. If Hallett were correct, A. Square could not understand the content of Desargues' theorem. However, it is hard to see why we should not think that A. Square can grasp what is a Desargues configuration of points and lines in the plane and even try to prove the theorem. ${ }^{42}$ This attempt will fail, provided that the investigation is restricted to projective means. But we see no reason to infer from that failure that the Flatlander failed to understand the theorem. As we see it, the Flatlander's grasp of the informal content of Desargues' theorem is sufficient for her having understood it.

We can make the point more generally. Since Desargues' theorem is stated in planar terms, it can be distinguished from other statements by investigators who only understand planar considerations. This ability to distinguish a statement from other statements is sufficient for a well-intentioned attempt to prove that statement, even if such attempts may be doomed to failure in particular cases. Such ability is typical of what we consider "basic" or "ordinary" understanding of mathematical statements, and this ability is plainly widespread, for instance among mathematical novices.

The previous discussion was limited to statements (such as Gödel sentences, the ParisHarrington statement, Desargues' theorem) whose inferential role is well understood. In elementary number theory there are statements for which nonelementary methods seem necessary for proof (think of Gödel sentences or Paris-Harrington). According to Hallett and Isaacson these statements would not be understandable by agents unfamiliar with nonelementary mathematics. We have already indicated our qualms about Hallett's position on this matter. However, this also invites reflection on a broader problem: what are we to say about statements whose inferential role is still unclear? How do we determine the content of a statement when we do not know for a fact whether it can be proved by elementary means? Consider again statements of elementary number theory for which nonelementary methods provide the only proofs known at present (think of Fermat's Last Theorem); and in addition statements like the Goldbach conjecture for which no proof or counterexample is presently known. It would seem, as a consequence of Hallett and Isaacson's proposal, that without a preliminary investigation of the status of the proposition (provable, disprovable or underivable in PA) we are not able to grasp what their content is. But that is certainly counterintuitive and, we claim, false. ${ }^{43}$

In reply, Hallett could agree with this point, while maintaining that a "complete" or "deeper" understanding of Desargues' theorem requires grasping its tacit spatial content, even if a "basic" or "ordinary" understanding does not. On this reply, the Flatlander could

42 Russell comments on Flatland in a discussion of the projective unprovability of Desargues' theorem by planar means; cf. Russell (1990a, p. 344).

43 For discussion of this and related points, cf. Arana (2008, §3). In a paper criticizing Dummett/Prawitz-style proof-theoretic semantics, Peter Pagin has made closely related points (cf. Pagin, 2009, pp. 19-20). Pagin argues that Dummett/Prawitz-style proof-theoretic semantics comes into conflict with the assumption of a compositional semantics. This is evident in the cases we are considering: for instance if Desargues' theorem has tacit spatial content on account of its inferential role within Hilbert's geometry, as Hallett would have it, it does not necessarily follow that any of the terms from which Desargues' theorem is composed, such as "triangle," has spatial content. 
understand Desargues' theorem, but only partly. A full understanding would only be achievable through understanding the inferential role of the statement, that is, through grasping its formal content, and not merely by grasping its informal content.

In reply, we note that grasping the formal content of statements may provide such a "deeper" type of understanding, but observe that measuring what is pure based on what belongs to the formal content of theorems threatens to trivialize purity, in making it the case that every theorem has a pure proof, when the content of that theorem is fully understood. Let us consider the cases studied by the two advocates of formal content under consideration, Hallett and Isaacson. We have seen that Hallett claims that Desargues' theorem has a pure proof on this construal of purity. In Isaacson's case, the Gödel sentences under consideration have nonarithmetical content; hence if we were to develop a measure of arithmetic purity based on Isaacson's construal of arithmetical content, Gödel sentences would have pure proofs as well. ${ }^{44}$ Both Desargues' theorem and Gödel sentences are cases where naively one would think that purity is impossible, since the gap between the expressive resources employed in each sentence and what is used in proving each sentence is so large. But on construing content formally, we are supposed to see that purity is in fact assured. If that were the case generally, so that every theorem has a pure proof when its formal content is revealed, then purity would cease to be an interesting property of proof. ${ }^{45}$ Since purity remains of interest today to mathematicians aware of these limitative results (for evidence, cf. Detlefsen \& Arana, 2011, §2), we maintain that this is not so. This, then, is a case for construing purity ascriptions in terms of informal rather than formal content (cf. Detlefsen \& Arana, 2011, §3.4 and §5).

Another point against formal content as the notion of content relevant to purity ascriptions is the following. We are normally willing to grant that the content of statements depends somewhat on the context in which they are made. Formal content entails, however, a radical contextualism regarding the content of statements like Desargues' theorem. The inferential role of Desargues' theorem within metrical geometries is quite different than its inferential role within projective geometry; in the former, spatial considerations are unnecessary, while in the latter they are necessary. In the setting of Hilbertian geometry, Desargues' theorem has tacit spatial content when used within a theory with axioms I.1, I.2,

44 We are only pushing this hypothetical possibility to spell out the analogy that Hallett finds between his own position and Isaacson's view on content. However, we should point out that Isaacson would resist tying purity to this notion of deeper content (personal communication).

45 Marcus Giaquinto has pressed on us the possibility in principle that there might be a theorem that is planar in formulation and has a solid proof but no planar proof, but at the same time has no role as a substitute for explicitly solid axioms. Such a statement would have neither informal nor formal solid content. We grant the possibility of such a case but point out that the trivialization we allege vitiates Hallett's notion of purity would still be present in such a case. Indeed, as soon as an axiomatic analysis of the theorem in question, let us call it $\mathrm{Q}$, is given, that analysis will tell us exactly what tools, beyond the planar projective axioms, are needed to prove Q. And since the formal content of $\mathrm{Q}$ will now be spelled out in the terms provided by the axiomatic analysis, Q will automatically have at least one pure proof. By the way, there is an explicit example of a situation similar to the one envisaged by Giaquinto, but in the context of an extension of the planar incidence axioms by axioms of congruency. If we extend the planar incidence axioms with the congruency axioms we can prove Desargues' theorem (by Menelaus' theorem, as pointed out earlier). However, Desargues' theorem does not imply any of the congruency axioms, because congruence cannot be defined in terms of incidence in Euclidean geometry, as Victor Pambuccian has pointed out to us. 
and III. ${ }^{46}$ However, consider now those axioms I.1, I.2, and III augmented by the axioms IV of congruence. Construing content formally entails that the content of Desargues' theorem changes dramatically depending on which axiomatic context we use it in, without its formulation changing at all. In the first axiomatic context, the formal content of Desargues' theorem would be spatial, whereas in the second axiomatic framework, it would not. ${ }^{47}$ In general, this conception of content will have as a consequence that no single content can be singled out as privileged but rather that each statement would be inhabited by a multiplicity of contents. This seems unpalatable.

It should be pointed out however that we are not claiming that the content of a statement is always independent of other statements. We deny this. All we need for our claim is that this is not always so and that it is not so for this particular case: the planar nature of the statement of Desargues' planar theorem cannot shift so radically between the two axiomatic frameworks.

One further point against construing content formally in purity ascriptions, as Hallett does, is that Hallett infers that the spatial content "revealed" by Hilbert's work belongs specifically to Desargues' theorem, when Hilbert's work shows only that Desargues' theorem added to the planar axioms of classes I and II (and in the Grundlagen, I, II, and III) has the same spatial consequences as the spatial axioms of those classes. Even if it is reasonable to maintain that the planar axioms plus Desargues' theorem have tacit spatial content on account of their shared inferential role (which we have contested), it is illicit to single out that content as belonging to Desargues' theorem. For those spatial consequences belong only to the axiomatic system as a whole, not to Desargues' theorem alone. While it is true that without Desargues' theorem these spatial consequences are not ensured, it is also true that Desargues' theorem alone does not ensure them. Hence it would be more accurate to say that these spatial consequences are partly the result of the planar axioms and partly the result of Desargues' theorem. Indeed, Hallett's argument would just as well establish that one of the planar axioms, say I.1, has tacit spatial content.

4.6. Desargues' theorem and metrical geometry. We have just offered a series of considerations against construing purity ascriptions in terms of formal content. If we are to take purity ascriptions from mathematical practice seriously, we have made the case that they must be construed in terms of informal content. Nevertheless, informal and formal content are intimately connected, as we indicated earlier: our grasp of the latter informs our grasp of the former, even if it does not replace it. In this section we thus change gears and take seriously the claim that statements of mathematics can have tacit formal contenteven though this does not change our approach to purity—revealed by investigation of their inferential role. We consider in particular the possibility that Desargues' theorem in fact has tacit metrical formal content, by investigating further what is shown by Hilbert's result on the equivalence of Desargues' theorem and the embeddability of planes in space.

In the Grundlagen, Hilbert stressed the relationship between Desargues' theorem and metrical considerations. Indeed, the title of Section \$23, in which Hilbert proves Theorem 33, is "The Impossibility of Demonstrating Desargues' Theorem for the Plane Without the Help of the Axioms of Congruence." In a recent paper (Gandon, 2004) Sébastien

46 Or I.1, I.2, II, and III, modulo the discussion in the appendix on order in projective geometry.

47 It is actually an open question what the exact formal content of Desargues' theorem is in the second axiomatic framework. However, for our needs it is only important that no spatial axioms are involved. 
Gandon has brought attention to a letter of Russell to Couturat from 1900 that builds on this relationship between Desargues' theorem and metric considerations. As Gandon explains (p. 209), Russell argues that because a particular geometrical calculus (due to Grassmann) can prove Desargues' theorem by purely planar means, this calculus must have introduced a consideration foreign to projective geometry, and in particular introduced a metric consideration. ${ }^{48}$ Thus for Russell, the ability to prove Desargues' theorem without appeal to spatial considerations is a sufficient condition for a theory's having metric content, and hence for not being purely projective.

That metrical matters are closely related to Desargues' theorem is plain from our earlier observation that by enabling the proof of the fourth harmonic point in von Staudt's quadrilateral construction, Desargues' theorem enables the coordinatization of projective geometry and even permits the development of the cross-ratio in projective terms. Thus Desargues' theorem has what may be reasonably viewed as metrical consequences, even if those metrical consequences may be carried out in what seem to be purely projective terms. Indeed, this is why von Staudt's work was judged so important by geometers.

We thus raise the following question: what explains the necessity of spatial considerations for proving Desargues' theorem in a purely projective way? Or to put it a different way: why does adding more dimensions to the plane permit the purely projective proof of Desargues' theorem? Hallett sees Hilbert as having answered this by proving that Desargues' theorem is necessary and sufficient for a plane's being embeddable in a space. Hallett infers from this and his theory of content that Desargues' theorem has tacit spatial content, and concludes that spatial considerations are therefore entirely appropriate in proving it. This does not answer the question in a fully satisfactory way, though. What grounds Hallett's claim that Desargues' theorem has tacit spatial content are simply Hilbert's results, rather than the identification of a feature of Desarguesian planes that makes clear why such planes are "well-behaved" enough to be embeddable in space. Hilbert's proof of Theorem 35 goes by way of the construction of an algebra of segments corresponding to Desarguesian planes, for which he shows that without the satisfaction of Desargues' theorem the desired coordinatization permitting embedding of the plane into space would not go through. But this leaves open the question of why this algebraic fact should have the geometrical consequences that it does. The remarkable coincidence of algebraic and geometric facts that Hilbert uncovered, and the central role of Desargues' theorem in this coincidence, remain unexplained.

Let us make this point more precise. The eminent algebraist Marshall Hall put the question as follows (cf. Hall, 1943, pp. 232): "Why is it that projective spaces of three or more dimensions are sufficiently regular to be coordinatized by skew-fields while projective nonDesarguesian planes exist which cannot be so coordinatized?" He suggests answering this question by looking carefully at the proof of the spatial Desargues theorem. He observes (pp. 232-233) that in this proof (given above on p. 312), each two of the three intersection points of the corresponding triangle sides determines a line, and the "kernel of the proof" is that these three lines can be identified with the line determined by the intersection of the

48 Russell to Couturat, dated June 21, 1900 (cf. Schmid, 2001, p. 182). The relevant passage from the letter is the following: “L'algèbre de Grassmann (même avant la multiplication) n'est pas celle de la géométrie projective toute pure: l'intensité y est essentielle, mais si elle ne représente pas une masse, elle ne saurait représenter qu'une idée métrique. C'est pour cette raison que Whitehead, qui est d'accord avec moi en ceci, parle de géométrie descriptive, non projective. Vous verrez qu'il prouve la construction de v. Staudt sans la troisième dimension (p. 215), ce qu'il ne saurait faire par une méthode vraiement projective." 
two planes in which the two triangles lie, and hence identified with each other as well. He observes that this is not necessarily the case in the plane. This makes more explicit how solid geometry differs from purely planar geometry. As Hall puts it:

In a space configuration of three or more dimensions the identification of a line, constructed as the intersection of two planes, with a line, constructed as the union of two points forces the identification of further constructed elements and the establishment of non-trivial configurations such as the Desargues configuration, while in the plane a line may be constructed only as a union of two points, a point as the intersection of two lines and there are no forced identifications. ${ }^{49}$

Hall identifies solid geometry as imposing constraints on what are permitted as lines that are not imposed by planar geometry. Though Hall does not offer any further explanation of why space and planes differ in this fundamental way, such a further explanation is possible, as we will now show.

A possibility is to look for such explanation in metrical terms. Klein and Peano, as we saw earlier, elaborated such an explanation for the restricted case of Riemannian geometries. The situation there was that planes could have nonconstant curvature, and in such geometries the geodesics will not lie on straight lines (as shown by Beltrami's theorem), and so if we take geodesics to be the lines, Desargues' theorem will fail. The existence of such "bad" planes is consistent with the assumptions of purely planar incidence geometry. Because Desargues' theorem is provable in spatial incidence geometry, such "bad" planes cannot exist in spatial incidence geometry. Thus we have a metrical characterization of these "bad" planes, and an intuitive explanation of why in space such planes cannot exist, in the case when the geometries under investigation are Riemannian. A next step would be to give such an explanation for more general classes of metrics/geometries.

Hilbert instigated such an investigation, in the fourth problem of his famous address to the International Congress of Mathematicians in Paris in 1900 (and thus essentially contemporaneously with the geometrical work we have been discussing). The title of the question is "problem of the straight line as the shortest distance between two points." Hilbert notes that the notion of a straight line has been defined by many as the shortest distance between two points; though he does not cite examples, he could have been thinking of Archimedes, Leibniz ${ }^{50}$ or Legendre, who wrote that "the straight line is the shortest path from one point to another" (cf. Legendre, 1794, definition III). ${ }^{51}$ Note that if such a definition were adopted, then since Desargues' theorem concerns straight lines, it would evidently have metrical content. As a result metrical considerations would not be

49 Cf. Hall (1943, p. 232). In proving his observation concerning the lack of "forced identifications" in the plane Hall invokes the notion of a free projective plane that will be discussed in Section 6 in the context of Joussen's work.

50 Cf. De Risi (2007, p. 226ff) for a discussion of Leibniz' interest in defining straight lines; though he endorsed a metrical definition he also considered nonmetrical definitions, as de Risi explains, and seems never to have settled on any one definition. Busemann (cf. Busemann, 1941) gives a brief development of the foundations of geometry in (what he takes to be) Leibniz's metrical setting.

51 A similar idea is also found in Poincaré: "That a line is straight is not a purely qualitative fact; one cannot ensure that a line is straight without making measurements, or without sliding over this line an instrument called a ruler, which is a sort of measuring instrument." Cf. Poincaré (1912, p. 483). 
impurities. Hilbert does not adopt such a definition; instead, he says that it ought to be thought a theorem. His fourth problem is to determine for what metrics this theorem holds, and to study the geometries resulting from such metrics. Busemann (cf. Busemann, 1976, p. 132) has observed that the problem had been solved by Beltrami's theorem for the case of Riemannian metrics, the only metrics studied by differential geometers up to the time of Hilbert's address. ${ }^{52}$ Thus, Busemann suggests, the fourth problem should be seen as Hilbert's attempt to widen the scope of investigation of metrical geometries, in particular of "the geometries in which the ordinary lines, i.e. lines of an $n$-dimensional (real) projective space $P^{n}$ or pieces of them are the shortest curves or geodesics" (cf. Busemann, 1976, p. 131).

In the 1900 address Hilbert described his interest in his fourth problem as follows:

... [B]ecause I believe that the thorough investigation of the conditions for the validity of this theorem will throw a new light upon the idea of distance, as well as upon other elementary ideas, e.g., upon the idea of the plane, and the possibility of its definition by means of the idea of the straight line, the construction and systematic treatment of the geometries here possible seem to me desirable. (cf. Hilbert, 1976, p. 416)

Hilbert's stated goal, then, was to further understanding of how metric matters bear on "elementary ideas" concerning, for example, the plane, by widening the domain of investigation of metrics/geometries for which the geodesics are "well-behaved," in the sense of behaving like projective straight lines. The relevance of Desargues' theorem is that it is a desirable property for straight lines to satisfy in a projective treatment of geometry: for as we have seen, its holding permits the development of von Staudt's projective coordinatization and development of cross-ratio via the quadrilateral construction. This is why Klein remarked that von Staudt's fusionist treatment "corresponds completely to the nature of the matter" (as quoted on p. 316 above). Hilbert's broadening of the question in his fourth problem does away with the implicit restriction to Riemannian geometries in Klein's time and permits a more general characterization of Desarguesian planes in terms of their metrical features. ${ }^{53}$ Busemann has formulated the problem in a quite general setting, ${ }^{54}$ and

52 Gerlich has observed that "It is a peculiarity of all classical plane geometries, namely the real projective plane, the real affine plane and the real hyperbolic plane that their lines are geodesics of a Riemannian metric. In this sense the classical incidence geometric structures are generated by differential geometric means." Cf. Gerlich (2005, p. 265).

53 Busemann observes that Desargues' theorem was central to Hilbert's proposed investigation; though Hilbert did not mention it explicitly in his address, Hamel, who completed his dissertation in 1901 under Hilbert's direction, did (cf. Hamel, 1903). In that text Hamel draws an explicit connection between Hilbert's aspiration in the fourth problem and Klein's work at p. 234.

54 Busemann defined the general notion of a " $G$-space," a general metric space where the extensions of geodesics are unique. He then defined a "Desarguesian space" of dimension $n$ as a $G$-space in which the geodesics are projective straight lines in real projective space of dimension $n$. Busemann proved that a necessary and sufficient condition for a $G$-space of dimension two (i.e., a plane) to be Desarguesian is that the geodesic passing through any two points is unique, and Desargues' theorem and its converse hold (cf. Busemann, 1955, §13, theorem 13.1). For $G$-spaces of dimension greater than two Desargues' theorem is no longer needed since the classical solid proofs go through in such settings (p. 65). There are non-Riemannian Desarguesian spaces, such as those given by Minkowski as described by Hilbert in the fourth problem. Riemannian Desarguesian spaces, and in particular planes, have constant curvature: this is Beltrami's theorem. Hilbert's fourth problem in this setting is to determine whether there is any other such characterization of Desarguesian planes, not restricting ourselves to Riemannian 
Pogorelov (cf. Pogorelov, 1979) is widely taken to have settled the problem so formulated, though due to the astonishing variety of metrics/geometries of which we are now aware, it is fair to say that no completely general solution to Hilbert's fourth problem is forthcoming (cf. Álvarez Paiva, 2005, p. 354). ${ }^{55}$

Such an investigation would plainly not be in the spirit of classical projective geometry. As Busemann observes of Hamel's work on Hilbert's fourth problem,

Hamel approached the problem through the Weierstrass Theory of the calculus of variations, which requires smoothness properties alien to the foundations of geometry, the framework visualized by Hilbert for the problem. (cf. Busemann, 1981, p. 87)

Our tentative suggestion is that an explanation of space's "homogenizing" or "smoothing" of planes so that planes embedded in space must be Desarguesian, while not otherwise, can be fruitfully pursued through an investigation of the metrical properties of planes and space, and their relationship. Indeed, in directing Hamel's investigation into his fourth problem, Hilbert seems to have endorsed such an investigation.

We close this section by clarifying why this type of metrical investigation would be valuable. In short, it would help explain the evident necessity of solid considerations for purely projective proofs of Desargues' theorem. We maintain that the notion of content relevant to purity ascriptions is what we have called informal content, rather than the notion of formal content on which Hallett argues the content of Desargues' theorem is tacitly spatial. However, we have tried to show in this section how Desargues' theorem is intertwined with not only spatial issues but also metrical issues, a matter of importance in understanding its formal content. Of particular importance is the search for metrical features of planes that ensure those planes are Desarguesian. The historical record reveals that there are two understandings of straight line: the understanding given by ordinary axiomatic geometry, and the metrical understanding as the path of shortest distance between two points. While these coincide in the standard Euclidean plane, nineteenth-century geometers had shown how they diverge on other surfaces, as we have seen. Hilbert advocated taking the ordinary axiomatic understanding as primary, and on that stance, metrical proofs of Desargues' theorem are impure, despite staying entirely within the plane. But if we were to adopt a metrical understanding of straight line, then metrical considerations become appropriate to Desargues' theorem and a case could then be made that metrical, purely planar proofs of Desargues' theorem (for instance, proofs using congruence by way of Menelaus' theorem) are pure. We are not advocating a metrical understanding of straight line, but rather observing that attempts to articulate the tacit content of Desargues' theorem should at least consider it.

\$5. Concluding thoughts. Consider the most prominent example of purity concerns in the foundations of mathematics: the elimination of geometrical considerations from the foundations of analysis and arithmetic in the work of Bolzano, Dedekind, and Weierstrass.

metrics. As Busemann expresses the problem in his terms, it is to characterize the Desarguesian spaces among all G-spaces with certain "nice" differentiability properties.

55 We observe that Hilbert's fourth problem, inasmuch as it focuses on geodesics that lie on lines in real projective spaces, is not general enough for a full explanation of the type we seek, since Hilbert's axioms for projective geometry are not restricted to real projective spaces, but characterize for instance complex and quaternionic projective spaces as a result of varying the base field. For such a study a quite general differential geometry would be needed. 
Their interest was not simply in identifying at which point one needs to appeal to geometrical considerations. Instead, their interest was normative: they believed that once identified these geometrical appeals must be eliminated. It would have been an unwelcome surprise to them if this had been impossible.

A similar stance is evident in the case of eliminating metrical considerations from projective geometry. The case for this elimination emerges from concerns regarding the use of congruence, similarity of triangles, proportions, order, and distance in projective geometry. For instance, von Staudt called for this elimination on grounds of generality, and as we have seen, his concern was normative: "geometric instruction must proceed from general considerations" [emphasis ours]. The fusionists we discussed in Section §2 mixed solid and planar considerations in their proofs of planar theorems, but they were still motivated by normative purity concerning the metrical in projective geometry. We saw as examples of this de Amicis' attempt to find proofs of planar results concerning just parallels and equality without using proportions but by using space instead (p. 309 above), and Lazzeri's attempt to eliminate the theory of proportions from theorems concerning similarity in the plane by means of spatial considerations (p. 310 above).

In the case that has been our central concern in this paper, however, the use of solid considerations in proving statements concerning the plane, the sort of purity that arises has a different feel. It is true that there were normative claims regarding planar geometry: recall Palatini's excoriation of mathematicians who were so "soft when it came to the distinctions between properties that belong to different theories within planimetry would want to enslave, so to speak, planimetry to stereometry" (p. 307 earlier). ${ }^{56}$ This is an exception, though; by contrast with the metrical/projective case we do not find a general normative concern for planar purity, wherein one calls for the elimination of solid considerations in proving planar statements. Given the recognition that metrical considerations can be avoided by going to space (through von Staudt's development of a projective coordinate system without explicit appeal to proportion, distance, or congruence, and his, Pasch's and Pieri's definition of a projective order via the theory of separation), and the realization that Desargues' theorem is intimately connected to the passage from planar to solid geometry (through the work of Hilbert), the kind of purity that is sought in the plane/solid case is more descriptive than normative. The interest was in identifying what could be done without appeal to space, while using only projective rather than metrical considerations.

Philosophically speaking, though, the difference between the normative and descriptive character of attitudes toward purity at play in this work is less interesting than the fact that once one realizes the role that Desargues' theorem plays concerning the embeddability of planes into space, then issues of content, in particular concerning planar and solid statements, arise. The question of whether Desargues' theorem, which seems to concern just the incidence and intersection of lines and points in the plane, is a statement of planar geometry, as would seem the default view, or a statement of solid geometry on account of

56 Palatini's view (discussed on p. 307 earlier) was that planar geometry is self-contained, requiring no "foreign" or "external" help. If we identify Palatini's system of planar geometry with Tarski's axioms for plane geometry in Tarski (1959), then we can cash out Palatini's view in terms of the logical completeness of Tarski's system (Tarski's Theorem 2). It follows from its completeness that every planar theorem expressible in Tarski's system has a planar proof. In this sense planar geometry is, as Palatini held, self-contained. However, Tarski's axioms define a ternary betweenness relation and a quaternary equidistance relation; arguably in virtue of the former and certainly in virtue of the latter, Tarski's geometry is metrical. Thus Palatini is correct that planar geometry is self-contained, but at the cost of using metrical considerations. 
its "tacit" or "hidden" spatial content, becomes urgent. Note that this issue is a precondition of pursuing normative purity projects for planar and solid geometry, for if seemingly planar statements like Desargues' theorem are really spatial in content, then it is senseless to try to eliminate solid considerations from their proofs.

We have argued here against Hallett's case that the classical solid proofs of Desargues' theorem are pure on account of the theorem having tacit spatial content. This case rests on a construal of content that implies, unhappily, that ordinary practitioners can fail to understand statements despite their evidently possessing all the conceptual resources required for such understanding. More precisely, we differentiated between the "informal" and "formal" content of statements, on which ordinary practitioners typically only have the conceptual resources to understand the former, while the latter is only understood by practitioners who have engaged in the kind of foundational investigations that Hilbert undertook for Desargues' theorem, and that Gödel undertook for Gödel sentences. We argued that Hallett's view of purity rests on construing content formally, and that this construal leads to problems in conceiving of purity ascriptions as they arise in mathematical practice. It threatens to trivialize purity, by suggesting that when purity seems impossible it is just because we have not fully understood the content of the statements in question, and that when we reach such fuller understanding we will see that the apparent impurity is not an impurity at all. In addition, Hallett's view of content involves an unwelcome radical contextualism of content depending on the axiomatic theory to which that statement belongs, and furthermore cannot locate the tacit spatial content specifically in Desargues' theorem as it claims, but only in the entire axiomatic system in which the statement is investigated.

Our view has been that there is a difference between statements of plane and solid geometry, and that this difference is one we can grasp at the level of "understanding" of the statements in question. This notion of "understanding" is taken as basic, although we do not exclude that it might be explicated through more detailed analytical work. Obviously, as we argued in Section 4.5, the problem of "content" can only be addressed if the primitive terms of our system are endowed with some appropriate interpretation. However, note that on our view the determination of whether a statement has planimetric or stereometric content cannot be reduced to a parsing of the (meaningful) vocabulary that goes into the statement in question. For instance, if we have a basic predicate $L$ ("lies on") and constants $a$ for a point and $B$ for a plane, the two statements $\neg \neg(a L B)$ and $\neg(a L B)$ have exactly the same logical and nonlogical vocabulary, but only the second purports to express a geometrical fact that requires an ambient space outside plane $B$. Thus, no matter which axiomatization of geometry is our focus (in particular whether the axiomatization takes only points as the basic category of objects, or has, possibly in addition to or instead of points, other basic categories), once we can reasonably provide the statements with an ordinary geometrical meaning, we have satisfied the conditions of possibility for a meaningful investigation of our problem.

A more radical objection consists in pointing out that in analytical investigations our distinctions become moot. We reply as follows. Cartesian analytic approaches take metrical notions for granted (for instance, the distance between two points is definable from the outset by a formula in the language), and so the necessity of solid considerations for planar statements like Desargues' theorem does not arise. Indeed, the analytic framework obliterates the distinction we are trying to articulate, but that only shows that important geometrical distinctions are lost once we move to analytic geometry.

Finally, let us summarize our position on the content of the statement of Desargues' theorem and our corresponding assessments on the purity, or lack thereof, of its proofs. 
We agree with Hilbert that the statement has "planar" content and thus we consider the proofs going through space to be impure. We thus take what we have called the "informal" content of a statement to be the notion of content relevant to purity ascriptions, and have argued against the relevancy of "formal" content in understanding such ascriptions. We suggested, though, a further investigation of the formal content of Desargues' theorem in which its formal content might in fact be metrical. Such an investigation requires a solution to the problem of whether the notion of straight line is metrical or not. We rest satisfied with posing this problem for further investigation.

§6. Appendix: order in projective geometry. Hallett characterizes Hilbert's investigations in both the lectures and in the Grundlagen as concerning "elementary projective geometry." These investigations include order axioms, but order is not clearly a projective notion, however. This poses a problem for Hallett, because he wants to argue that Hilbert's work shows that solid proofs of Desargues' theorem are pure, but if order does not belong to projective geometry, then Hilbert's proofs of projective theorems using order are evidently impure. This problem is particularly clear for the case of Desargues' theorem, since it concerns only the incidence and intersection of lines and points, and not any order-theoretic properties of configurations. Hallett's claim that solid proofs of Desargues' theorem are pure would thus be threatened by appeal to order axioms. ${ }^{57}$ Recent work shows, however, that order axioms are not needed for proving Desargues' theorem in Hilbert's axiomatic framework, from which it follows that Hilbert's results go through in a less controversially projective setting. In this appendix we explain how these issues are resolved.

The axioms of Hilbert's class II implicitly define what it is for a point collinear with two other points to be "between" those two other points. Hilbert shows that these axioms can be used to generate an order on points on lines, thus justifying his naming them axioms of order. Hilbert includes order axioms in his investigations into Desargues' theorem, in both the 1898/1899 lectures and in the Grundlagen. In the 1898/1899 lectures, Hilbert writes that "Axioms I, II formed essentially the foundation [Grundlage] of projective geometry." 58 Schur, who Hilbert credited in 1898 for reinvigorating his interest in the foundations of geometry (cf. Toepell, 1986a, 1986b, p. 338), writes that "We understand by projective axioms those which Hilbert called axioms of connection [Verknüpfung] and order," that is, Hilbert's axioms I and II (cf. Schur, 1901, p. 267).

While Hilbert's incidence axioms I are uncontroversially projective, the same cannot be said for his order axioms II. The issue concerns how membership in projective geometry

57 A possible defense available to Hallett would be Coxeter's suggestion (cf. Coxeter, 1989, p. 176) that straight lines are rightly defined in terms of betweenness: a line segment is, by definition, the set of points between two points. As Coxeter puts it, "The essential idea is intermediacy (or 'betweenness'), which Euclid used in his famous definition: A line (segment) is that which lies evenly between its ends. This suggests the possibility of regarding intermediacy as a primitive concept and using it to define a line segment as the set of all points between two given points." Coxeter then defines rays in terms of line segments and betweenness, and lines in terms of rays and line segments. However, this approach rests on a peculiar translation of Euclid's definition I.4, and has not been widely adopted. Cf. Arana, (2009, pp. 4-5), for discussion of Coxeter's definition and its context; and Pambuccian (2009) for a foundational investigation concerning Coxeter's definition.

58 Cf. Hallett \& Majer (2004, p. 343). Earlier in the lectures he also makes this point (p. 319), adding that Axioms I and II are only "essentially" the foundation of projective geometry, but because some continuity assumptions are also needed, not because of order. 
ought to be construed. A typical construal of projective geometry is that it is the study of projective invariants. However, the basic property axiomatized by axiom class II, the ternary relation of betweenness, is not a projective invariant (cf. Seidenberg, 1962, §7). Hence on this construal of projective geometry, it would seem problematic to include order in a projective investigation.

Russell, for one, took this view. In the Principles of Mathematics he writes (Russell, 1903, p. 393) that "it seems improper to include in projective Geometry any property which is not unaltered by projection," and observes "the elementary sense of between... is in general not unaltered by projection." In a private notebook he puts it even more starkly: "Projective Geometry is not essentially concerned with order or series" (cf. Russell, 1990b, p. 379). ${ }^{59}$ This view is quite standard; for instance, Veblen and Young remark in the first of their two-volume text Projective Geometry that "projective geometry is concerned with those properties of figures that are left unchanged when the figures are subjected to a projective transformation" (cf. Veblen \& Young, 1916, p. 14), and that since "linear order" is an element of "the theory of functions of a real variable rather than of elementary geometry" (p. iii), they will leave out any discussion of order from the first, more elementary volume.

Poincaré too seems to have taken this view. In his review of Hilbert's Grundlagen, Poincaré translates the name of Hilbert's axioms I, the axioms of "Verknüpfung," as the "projective axioms" [axiomes projectifs] (cf. Poincaré, 1902, p. 254). In excluding the axioms II of order in this naming we may suppose Poincaré did not consider the axioms of order to be projective.

It thus remains to be explained why Hilbert included order axioms in the "foundation" of projective geometry. Hilbert gives a clue by citing Pasch's Vorlesungen über Neuere Geometrie (Pasch, 1882) as he explains his axioms of order in the Grundlagen. Pasch's treatment of the foundations of geometry begins with a general treatment of geometry, and then turns specifically to a development of projective geometry. ${ }^{60}$ The general treatment includes order axioms, specifically for the ternary relation of betweenness. He then shows how to develop projective geometry specifically, using as a primitive not betweenness (evidently recognizing its projective variance) but rather the quaternary relation of "separation" of points in a harmonic range. ${ }^{61}$ The separation relation enables a projective treatment of order by yielding an ordering between pairs of points, though as a result it will not have all the same properties as the ordinary ternary betweenness relation. ${ }^{62}$ It is straightforward to show that this relation is a projective invariant, and so Pasch's treatment of projective geometry meets the criterion of studying just projective invariants.

Because the separation relation is quaternary, Hilbert's axioms II cannot be understood as axiomatizing this relation, since the betweenness relation it axiomatizes is tertiary. We propose, however, that Hilbert's inclusion of order axioms in the "foundation" of projective

59 Cf. Gandon (2009) for more on the nineteenth-century debate about whether order belongs to projective geometry, especially concerning Russell's role in this debate.

60 Cf. Schlimm (2010) for a fuller discussion of Pasch's foundational methodology.

61 Cf. Russell (1903, §383-\$387, pp. 400-403) for a description of how Pasch develops projective geometry from his general foundations of geometry (which Russell calls "Descriptive Geometry").

62 Shortly thereafter, Pieri showed how the separation relation can be defined purely in terms of incidence properties, though additional axioms are needed to ensure that the resulting order has the usual properties of an order. Cf. Whitehead (1906, chap. IV) for further details on this development, and pp. 24-26 for a definition of separation; and Marchisotto \& Smith (2007, pp. 128-137) for details on Pieri's role in this development. 
geometry reflects Pasch's attitude concerning the foundations of geometry. Hilbert does not mean that betweenness itself belongs to projective geometry, but rather that it can, following Pasch, be used to develop projective geometry, which can be shown to include an ordering (though not the one axiomatized by II).

We need not fully resolve this tricky interpretative issue, since our main interest is in Desargues' theorem, which concerns just the incidence and intersection of lines and points and says nothing about the order of those points on those lines. As it turns out, order axioms are not needed for proving Desargues' theorem in Hilbert's axiomatic framework. Hilbert's incidence axioms I together with his parallel axiom III suffice for proving the affine formulation of Desargues' theorem (as stated in the Grundlagen). Hilbert's incidence axioms I together with an axiom stating that there are (at least) three points on every line (which Hilbert did not include in his axioms, but was included as Axiom E0 in Veblen \& Young, 1916, p. 18) suffices for proving the projective formulation of Desargues' theorem (as stated in the 1898/1899 lecture notes); without this additional axiom, the incidence axioms I do not suffice, as shown in Hartvigson (1974). In Joussen (1966) it is shown that order axioms do not enable the planar projective proof of even any special cases of Desargues' theorem, by showing that ordered projective planes do not satisfy any such "interesting" configuration theorems. ${ }^{63}$

Moreover, order axioms are not needed to prove the sufficiency of (the projective formulation of) Desargues' theorem for the satisfaction of the spatial incidence axioms in the presence of axioms of planar projective geometry. Plane projective geometry can be expressed by Hilbert's planar incidence axioms I 1-2 plus a "projective" axiom asserting that any two lines intersect, or by Hilbert I 1-2 plus the "Veblen-Young axiom" that for any four points $a, b, d, c$ in the plane such that no three are collinear, if the lines $a b$ and $c d$ do not intersect, then neither do the lines $a c$ and $b d .{ }^{64}$ That the satisfaction of Desargues' theorem is sufficient for the embeddability of planes satisfying the axioms of planar projective geometry was proved by Levi (cf. Levi, 1939), by showing that a geometry satisfying the axioms of planar projective geometry plus the projective Desargues' theorem can be embedded in a spatial projective geometry (all of I plus the projective axiom), and indeed that Desargues' theorem is necessary and sufficient for this embeddability. The difference with Hilbert's Theorems 33 and 35, again, is that Levi does not involve order axioms in his results. Similarly, the affine formulation of Desargues' theorem in the Grundlagen can be shown to be necessary and sufficient for the embeddability of planar affine geometry (that is, Hilbert's planar incidence axioms I 1-2 plus the parallel axiom III) in a spatial affine geometry (all of I plus III).

63 Specifically, Joussen showed that the "free projective plane" is orderable; cf. Hall $(1943, \S 4)$ for a precise definition of free projective plane. Kalhoff (1988) gives a precise characterization of exactly what order contributes to projective planes, using the algebraic notion of a "formally real ternary field."

64 The Veblen-Young axiom is a purely incidence-based formulation of "Pasch's axiom" (Grundsatz IV, Pasch, 1882, p. 21), which was stated in Peano (1894) in the two postulates XIII and XIV (p. 64), and by Hilbert in the Grundlagen as Axiom II.5. Pasch's formulation is order-theoretic, stated in terms of betweenness; Peano and Hilbert follow Pasch in this regard. The axiom was weakened in Moore (1902) as Axiom 4 (p. 147) and weakened further in Veblen (1904) as Axiom VIII (p. 345), though both of these weakenings remain order-theoretic (the latter uses an ordering on the points of a line, while the former uses the notion of the "interior" and "exterior" of a triangle). In Veblen \& Young (1916) the reference to order is removed, though consequently the resulting axiom expresses that any two lines in the plane meet, unlike the order-based formulation of Pasch and the others. This projective requirement, though, is just what we are looking for here. 
Finally, the relationship between models of Hilbert's I (i.e., without order assumptions) and projective spaces of dimension three can be clarified as follows. Models of Hilbert's I satisfying the axiom that there are at least three points on every line can be embedded into projective spaces of dimension three. This follows from Kahn's theorem (cf. Kahn, 1980), reproved as Theorem 5.2 in Kreuzer (1996, p. 97); again Hartvigson (1974) implies that the assumption that there are at least three points on every line is necessary.

§7. Acknowledgments Thanks to Fabio Acerbi, Kirsti Andersen, David Auckly, John Baldwin, Bruno Belhoste, Paola Cantù, Vincenzo De Risi, Philip Ehrlich, Paolo Freguglia, Sébastien Gandon, Marcus Giaquinto, Jeremy Gray, Angelo Guerraggio, Niccoló Guicciardini, Michael Hallett, Jeremy Heis, Dan Isaacson, Alexander Kreuzer, John MacFarlane, Elena Marchisotto, Sébastien Maronne, Philippe Nabonnand, Pier Daniele Napolitani, Victor Pambuccian, Marco Panza, Doug Patterson, Patrick Popescu-Pampu, David Rabouin, Steve Russ, Dirk Schlimm, Thomas Schneider, Ivahn Smadja, and Markus Stroppel. In addition we are very grateful for the extensive comments by the two anonymous referees, which greatly improved the paper. Lastly, the first author thanks the Agence Nationale de la Recherche, France, for its support during work on this project.

\section{BIBLIOGRAPHY}

Abbott, E. (1884). Flatland: A Romance of Many Dimensions. London: Seeley and Co.

Álvarez Paiva, J. C. (2005). Symplectic geometry and Hilbert's fourth problem. Journal of Differential Geometry, 690(2), 353-378.

Amaldi, U. (1900). Sulla teoria dell'equivalenza. In Enriques, F., editor. Questioni Riguardanti la Geometria Elementare. Bologna, Italy: Zanichelli, pp. 103-142.

Andriani, A., Angeleri, F., \& Giulio Lazzeri (1904). Note all'inchiesta sulla fusione della geometria piana con la solida. Periodico di Matematica, 19, 292-296.

Anonymous. (1901). La fusione della planimetria e stereometria in Francia. Bollettino della Mathesis, 50(4).

Arana, A. (2008). Logical and semantic purity. Protosociology, 25, 36-48. Reprinted in Philosophy of Mathematics: Set Theory, Measuring Theories, and Nominalism, Gerhard Preyer and Georg Peter (eds.), Ontos, 2008.

Arana, A. (2009). On formally measuring and eliminating extraneous notions in proofs. Philosophia Mathematica, 17, 208-219.

Archimedes. (2004). The Works of Archimedes, Vol. I. Cambridge, UK: Cambridge University Press. Translated into English with critical comments by Reviel Netz.

Audin, M. (2003). Geometry. Berlin: Springer-Verlag. Translated from the 1998 French original.

Avellone, M., Brigaglia, A., \& Zappulla, C. (2002). The foundations of projective geometry in Italy from De Paolis to Pieri. Archive for History of Exact Sciences, 560(5), 363-425.

Belhoste, B. (1998). De l'École Polytechnique à Saratoff, les premiers travaux géométriques de Poncelet. Bulletin de la Société des Amis de la Bibliothèque de l'École Polytechnique, 19, 9-29.

Belhoste, B., \& Taton, R. (1989). L'invention d'une langue des figures. In Dhombres, J., editor. L'École Normale de l'An III, Leçons de Mathématiques, Laplace-LagrangeMonge. Paris, France: Dunod, pp. 269-303.

Bellavitis, G. (1838). Saggio di geometria derivata. Nuovi Saggi della Imperiale Regia Accademia di Scienze Lettere ed Arti in Padova, 4, 243-288. 
Beltrami, E. (1902). Resoluzione del problema: riportare i punti di una superficie sopra un piano in modo che le linee geodetiche vengano rappresentate da linee rette. Annali di Matematica Pura et Applicata, 10(7), 185-204. Reprinted in Opere Matematiche di Eugenio Beltrami, Book 1, Ulrico Hoepli, Milano, 1902.

Bernays, P. (1922). Die Bedeutung Hilberts für die Philosophie der Mathematik. Die Naturwissenschaften, 10, 93-99. English translation in Paolo Mancosu. From Brouwer to Hilbert. Oxford University Press, New York, 1998. pp. 189-197.

Bettazzi, R. (1891). Review of G. Lazzeri e A. Bassani, Professori della R. Accademia Navale - Elementi di Geometria - Livorno. Tip. di R. Giusti, 1891. Periodico di Matematica, 6, 155-163.

Bkouche, R. (1991). Variations autour de la réforme de 1902/1905. In Gispert, H., et al., editors. La France Mathématique. Paris, France: Cahiers d'Histoire et de Philosophie des Sciences et Société Mathématique de France, pp. 181-213.

Bkouche, R. (1996). La place de la géométrie dans l'enseignement des mathématiques en France, de la réforme de 1902 à la réforme des mathématiques modernes. In Belhoste, B., Gispert, H., and Hulin, N., editors. Les Sciences au Lycée. Paris, France: Vuibert, Paris.

Bkouche, R. (2003). La géométrie dans les premières années de le revue L'Enseignement Mathématique. In Coray, D., Furinghetti, F., Gispert, H., Hodgson, B. R., and Schubring, G., editors. One Hundred Years of L'Enseignement Mathématique (Moments of Mathematics Education in the Twentieth Century). Genève, Switzerland: L'Enseignement Mathématique, pp. 95-113.

Bolondi, G., editor. (2002). La Mathesis. La Prima Metà del Novecento nella "Società Italiana di Scienze Matematiche e Fisiche”, Vol. 5 of PRISTEM/Storia. Milano, Italy: Springer.

Bolzano, B. (2005). The Mathematical Works of Bernard Bolzano. Oxford, UK: Oxford University Press. Edited by Steve Russ.

Bretschneider, C. A. (1844). Lehrgebäude der niederen Geometrie. Jena: F. Frommann.

Busemann, H. (1941). On Leibniz's definition of planes. American Journal of Mathematics, 630(1), 101-111.

Busemann, H. (1955). The Geometry of Geodesics. New York, NY: Academic Press Inc.

Busemann, H. (1976). Problem IV: Desarguesian spaces. In Browder, F., editor. Mathematical Developments Arising from Hilbert problems (Proc. Sympos. Pure Math., Northern Illinois Univ., De Kalb, Ill., 1974). Proc. Sympos. Pure Math., Vol. XXVIII. Providence, RI: American Mathematical Society, pp. 131-141.

Busemann, H. (1981). Review of Hilbert's Fourth Problem. Bulletin of the American Mathematical Society, 40(1), 87-90.

Candido, G. (1899). Sur la fusion de la planimétrie et de la stéréométrie. L’Enseignement Mathématique, 1, 204-215.

Carnap, R. (1937). Logical Syntax of Language. London: Kegan Paul, Trench, Trubner and Co. Translated by Amethe Smeaton (Countess von Zeppelin).

Cavalieri, B. (1635). Geometria Indivisibilibus Continuorum Nova Quadam Ratione Promota. Bologna, Italy: Clemente Ferroni.

Cerroni, C. (2004). Non-Desarguian geometries and the foundations of geometry from David Hilbert to Ruth Moufang. Historia Mathematica, 31, 320-336.

Chasles, M. (1837). Aperçu Historique sur l'Origine et le Développement des Méthodes en Géométrie. Bruxelles, Belgium: M. Hayez.

Courant, R., \& Robbins, H. (1996). What Is Mathematics? (second edition). New York: Oxford University Press. Revised by Ian Stewart. 
Coxeter, H. S. M. (1989). Introduction to Geometry (second edition). New York: Wiley.

Coxeter, H. S. M., \& Greitzer, S. L. (1967). Geometry Revisited. Washington, D.C.: Mathematical Association of America.

Cremona, L. (1873). Elementi di Geometria Projettiva. Roma, Italy: G.B. Paravia. Translated into French by Edouard Dewulf, Gauthier-Villars, Paris, 1875.

De Amicis, E. (1897-1898). Pro fusione. Relazione sulla questione V proposta nel N.1 (anno I) del Bollettino dell'Associazione Mathesis "Opportunità della fusione della geometria piana con la solida nell'insegnamento". Bollettino della Mathesis, 20(4), 73-96. Republished in Periodico di Matematica 13, 1898, pp. 49-72.

De Paolis, R. (1884). Elementi di Geometria. Torino, Italy: Loescher.

De Risi, V. (2007). Geometry and Monadology: Leibniz's Analysis Situs and Philosophy of Space. Basel: Birkhäuser.

Detlefsen, M. (2008). Purity as an ideal of proof. In Mancosu, P., editor. The Philosophy of Mathematical Practice. Oxford: Oxford University Press, pp. 179-197.

Detlefsen, M., \& Arana, A. (2011). Purity of methods. Philosophers' Imprint, 110(2), $1-20$.

Dhombres, J., editor. (1989). L'École Normale de l'An III, Leçons de Mathématiques, Laplace-Lagrange-Monge. Paris, France: Dunod.

Euclid. (2007). Tutte le Opere. Milano, Italy: Bompiani. Edited by Fabio Acerbi.

Gandon, S. (2004). Russell et l'Universal Algebra de Whitehead: la géométrie projective entre ordre et incidence (1898-1903). Revue d'Histoire des Máthematiques, 10, $187-256$.

Gandon, S. (2009). Toward a topic-specific logicism? Russell's theory of geometry in The Principles of Mathematics. Philosophia Mathematica, 170(1), 35-72.

Gergonne, J. (1825-1826). Philosophie mathématique. Considérations philosophiques sur les élémens de la science de l'étendue. Annales de Mathématiques Pures et Appliquées, 16, 209-231.

Gerlich, G. (2005). Topological affine planes with affine connections. Advances in Geometry, 50(2), 265-278.

Giacardi, L., \& Roero, C. S. (1996). La nascita della Mathesis (1895-1907). In Giacardi, L., and Roero, C. S., editors. Dal Compasso al Computer. Torino, Italy: Associazione Mathesis.

Giudice, F. (1899). Sulla fusione della geometria piana e solida. Periodico di Matematica, 14, 34-36.

Gray, J. J., \& Field, J. (1987). The Geometrical Work of Girard Desargues. New York, NY: Springer-Verlag.

Guggenheimer, H. (1974). The theorem of Menelaus in axiomatic geometry. Geometriae Dedicata, 3, 257-261.

Hall, M. (1943). Projective planes. Transactions of the American Mathematical Society, 54, 229-277.

Hallett, M. (2008). Reflections on the purity of method in Hilbert's Grundlagen der Geometrie. In Mancosu, P., editor. The Philosophy of Mathematical Practice. Oxford: Oxford University Press, pp. 198-255.

Hallett, M., \& Majer, U., editors. (2004). David Hilbert's Lectures on the Foundations of Geometry, 1891-1902. Berlin, Germany: Springer-Verlag.

Hamel, G. (1903). Über die Geometrieen, in denen die Geraden die Kürzesten sind. Mathematische Annalen, 57, 231-264.

Hartshorne, R. (1967). Foundations of Projective Geometry, Vol. 1966/67 of Lecture Notes, Harvard University. New York, NY: W. A. Benjamin, Inc. 
Hartvigson, Z. R. (1974). A non-Desarguesian space geometry. Fundamenta Mathematicae, 86, 143-147.

Heath, T. (1921). A History of Greek Mathematics, Vol. I. Oxford, UK: Clarendon Press. ISBN 0-486-24073-8.

Hilbert, D. (1899). Grundlagen der Geometrie. Leipzig, Germany: B.G. Teubner.

Hilbert, D. (1901). Mathematische Probleme. Archiv der Mathematik und Physik (3rd series), 1, 44-63, 213-237. English translation by M.W. Newson in the Bulletin of the American Mathematical Society, 8, 437-479.253-297, 1902.

Hilbert, D. (1905a). Logische Principien des mathematischen Denkens. Vorlesung, Sommersemester 1905. Lecture notes by Max Born. Unpublished manuscript, $277 \mathrm{pp}$. Niedersächsische Staats-und Universitätsbibliothek Göttingen, Handschriftenabteilung, Cod. Ms. D. Hilbert 558a.

Hilbert, D. (1905b). Logische Principien des mathematischen Denkens. Vorlesung, Sommersemester 1905. Lecture notes by Ernst Hellinger. Unpublished manuscript, 277 pp. Bibliothek, Mathematisches Institut, Universität Göttingen.

Hilbert, D. (1971). Foundations of Geometry. La Salle, IL: Open Court. English translation of Grundlagen der Geometrie (B.G. Teubner, Leipzig, 1899), by L. Unger.

Hilbert, D. (1976). Mathematical problems. In Browder, F., editor. Mathematical Developments Arising from Hilbert Problems, Vol. 28 of Proceedings of Symposia in Pure Mathematics. Providence, RI: American Mathematical Society, pp. 1-34. Translated by M.W. Newson. Originally published in 1900.

Hilbert, D. (1992). Natur und mathematisches Erkennen. Basel, Switzerland: Birkhäuser. Vorlesungen, 1919-1920.

Hilbert, D., \& Cohn-Vossen, S. (1952). Geometry and the Imagination. New York, NY: Chelsea Publishing Company. Translated by P. Neményi.

Hon, G., \& Goldstein, B. (2008). From Summetria to Symmetry: The Making of a Revolutionary Scientific Concept. Dordrecht: Springer.

Isaacson, D. (1996). Arithmetical truth and hidden higher-order concepts. In Hart, W. D., editor. The Philosophy of Mathematics. New York: Oxford University Press, pp. 203224. First published in Logic Colloquium '85, the Paris Logic Group (eds.), Amsterdam: North-Holland, 1987, pp. 147-169.

Joussen, J. (1966). Die Anordnungsfähigkeit der freien Ebenen. Abhandlungen aus dem Mathematischen Seminar der Universität Hamburg, 29, 137-184.

Kahn, J. (1980). Locally projective-planar lattices which satisfy the bundle theorem. Mathematische Zeitschrift, 1750(3), 219-247.

Kalhoff, F. (1988). Eine Kennzeichnung anordnungsfähiger Ternärkörper. Journal of Geometry, 310(1-2), 100-113.

Klein, F. (1873). Über die sogenannte Nicht-Euklidische Geometrie. Mathematische Annalen, 6, 112-145.

Klein, F. (1953). Elementary Mathematics from an Advanced Standpoint. Geometry. New York, NY: Dover. Translated from the third German edition by E. R. Hedrick and C. A. Noble, Macmillan, New York, 1939.

Knorr, W. R. (1993). The Ancient Tradition of Geometric Problems. New York, NY: Dover Publications. Corrected reprint of the 1986 original.

Kreisel, G. (1980). Kurt Gödel. Biographical Memoirs of Fellows of the Royal Society, 26, 149-224.

Kreuzer, A. (1996). Locally projective spaces which satisfy the bundle theorem. Journal of Geometry, 560(1-2), 87-98. 
Lazzeri, G. (1899). Note alla discussione della prima questione trattata dal congresso. Periodico di Matematica, 14, 117-124.

Lazzeri, G. (1904). A proposito dell'inchiesta fatta dall'associazione Mathesis sulla fusione della geometria piana con la solida. Periodico di Matematica, 19, 233-240.

Lazzeri, G., \& Bassani, A. (1898). Elementi di Geometria (second edition). Livorno, Italy: Raffaello Giusti. First edition 1891.

Legendre, A.-M. (1794). Élements de Geometrie. Paris, France: Firmin Didot.

Levi, F. M. (1939). On a fundamental theorem of geometry. Journal of the Indian Mathematical Society, 3-4, 82-92.

Loria, G. (1899). La fusione della planimetria con la stereometria (una pagina di storia contemporanea). Periodico di Matematica, 15, 1-7.

Loria, G. (1905). Sur l'enseignement des mathématiques élémentaires en Italie. L'Enseignement Mathématique, 7, 11-20.

Manara, C. F., \& Spoglianti, M. (1977). La idea di iperspazio: Una dimenticata polemica tra G. Peano, C. Segre, e G. Veronese. Memorie della Accademia Nazionale di Scienze, Lettere e Arti, di Modena (series 6), 19, 109-129.

Mancosu, P. (1996). Philosophy of Mathematics and Mathematical Practice in the Seventeenth Century. New York, NY: Oxford University Press.

Mancosu, P. (1998). From Brouwer to Hilbert. New York, NY: Oxford University Press.

Mancosu, P., editor. (2008). The Philosophy of Mathematical Practice. Oxford: Oxford University Press.

Mancosu, P., \& Arana, A. (2010). Descartes and the cylindrical helix. Historia Mathematica, 370(3), 403-427.

Marchisotto, E. A., \& Smith, J. T. (2007). The Legacy of Mario Pieri in Geometry and Arithmetic. Boston, MA: Birkhäuser Boston Inc. With a foreword by Ivor GrattanGuinness.

McCleary, J. (1982). An application of Desargues' theorem. Mathematics Magazine, 550(4), 233-235.

Méray, C. (1874). Nouveaux Eléments de Géométrie. Paris, France: Savy.

Möbius, A. F. (1827). Der Barycentrische Calcul. Leipzig, Germany: Johann Ambrosius Barth. Reprinted in Gesammelte Werke, Vol. 1, edited by R. Baltzer, S. Hirzel, Leipzig, 1885 , pp. $1-388$.

Monge, G. (1795). Leçons à l'École Normale de l'An III. In Dhombres, J., editor. L'École Normale de l'An III, Leçons de Mathématiques, Laplace-Lagrange-Monge. Paris, France: Dunod.

Moore, E. H. (1902). On the projective axioms of geometry. Transactions of the American Mathematical Society, 30(1), 142-158.

Moulton, F. R. (1902). A simple non-Desarguesian plane geometry. Transactions of the American Mathematical Society, 30(2), 192-195.

Pagin, P. (2009). Compositionality, understanding, and proofs. Mind, 118, 713-737.

Palatini, F. (1897-1898). Osservazioni sulla Nota "Pro fusione" del Prof. de Amicis. Bollettino della Mathesis, 20(5), 120-124.

Palatini, F. (1899). Una conversazione coi fusionisti. Periodico di Matematica, 14, 205-208.

Palmieri, P. (2009). Superposition: On Cavalieri's practice of mathematics. Archive for History of Exact Sciences, 630(5), 471-495.

Pambuccian, V. (2009). A reverse analysis of the Sylvester-Gallai theorem. Notre Dame Journal of Formal Logic, 500(3), 245-260.

Panza, M., \& Malet, A. (2010). Wallis on indivisibles. Draft. 
Pappus. (1876-1878). Collectionis Quae Supersunt. Berlin, Germany: Weidman. 3 vols. Edited and translated into Latin by F. Hultsch.

Pasch, M. (1882). Vorlesungen über neuere Geometrie. Leipzig, Germany: B.G. Teubner.

Peano, G. (1891). Osservazioni del Direttore sull'articolo precedente. Rivista di Matematica, 1, 66-69.

Peano, G. (1894). Sui fondamenti della geometria. Rivista di Matematica, 4, 51-90.

Peckhaus, V. (1990). Hilbertprogramm und kritische Philosophie. Göttingen, Germany: Vandenhoeck and Ruprecht.

Plücker, J. (1830). Über ein neues Coordinatensystem. Journal für die reine und angewandte Mathematik, 5, 1-36.

Pogorelov, A. (1979). Hilbert's Fourth Problem. Washington, DC: V. H. Winston \& Sons. Translated by Richard A. Silverman.

Poincaré, H. (1902). Review of Hilbert's Grundlagen der Geometrie. Journal des Savants, 252-271. Modification of an earlier version published in Bulletin des Sciences Mathématiques, vol. 26, 1902, pp. 249-272.

Poincaré, H. (1912). Pourquoi l'espace a trois dimensions. Revue de Métaphysique et de Morale, 200(4), 483-504.

Poncelet, J.-V. (1822). Traité des Propriétés Projectives des Figures. Paris, France: Bachelier.

Russell, B. (1903). The Principles of Mathematics. Cambridge, UK: Cambridge University Press.

Russell, B. (1990a). Note on Order [1898]. In Griffin, N., and Lewis, A. C., editors. Philosophical Papers 1896-99. London: Unwin Hyman.

Russell, B. (1990b). Notes on Geometry [1899]. In Griffin, N., and Lewis, A. C., editors. Philosophical Papers 1896-99. London: Unwin Hyman.

Sakarovitch, J. (1989). La géométrie descriptive après Monge. In Dhombres, J., editor. L'École Normale de l'An III, Leçons de Mathématiques, Laplace-Lagrange-Monge. Paris, France: Dunod, pp. 583-590.

Schlimm, D. (2010). Pasch's philosophy of mathematics. The Review of Symbolic Logic, 30(1), 93-118.

Schmid, A.-F., editor. Bertrand Russell/Louis Couturat (1897-1913); Correspondance sur la philosophie, la logique et la politique. Paris, France: Kimé.

Schneider, T., \& Stroppel, M. (2007). Automorphisms of Hilbert's non-desarguesian affine plane and its projective closure. Advances in Geometry, 70(4), 541-552.

Schur, F. (1901). Ueber die Grundlagen der Geometrie. Mathematische Annalen, 550(2), 265-292.

Segre, C. (1891). Su alcuni indirizzi nelle investigationi geometriche: Osservazioni dirette ai miei studenti. Rivista di Matematica, 1, 42-66.

Seidenberg, A. (1962). Lectures In Projective Geometry. Princeton: D. Van Nostrand.

Sperner, E. (1954). Ein gruppentheoretischer Beweis des Satzes von Desargues in der absoluten Axiomatik. Archiv der Mathematik, 5, 458-468.

Steiner, J. (1832). Systematische Entwickelung der Abhängigkeit geometrischer Gestalten von einander. Berlin: G. Fincke.

Stroppel, M. (2010). Early explicit examples of non-desarguesian plane geometries. Technical Report 2010-013, Stuttgarter Mathematische Berichte, Stuttgart. Forthcoming in the Journal of Geometry.

Tarski, A. (1959). What is elementary geometry? In Henkin, L., Suppes, P., and Tarski, A., editors. The Axiomatic Method. Amsterdam: North-Holland. 
Taton, R. (1951a). L'Oeuvre Mathématique de G. Desargues. Paris, France: PUF. Second edition with postface, J. Vrin, Paris, 1981.

Taton, R. (1951b). L'Oeuvre Scientifique de Monge. Paris: PUF.

Taton, R. (1951c). Découverte d'un exemplaire original du "Brouillon project" sur les Coniques de Desargues. Revue d'Histoire des Sciences et de Leurs Applications, 40(2), $176-181$.

Toepell. (1986a). Über die Entstehung von David Hilberts “Grundlagen der Geometrie”. Göttingen, Germany: Vandenhoeck and Ruprecht.

Toepell, M. (1986b). On the origins of David Hilbert's Grundlagen der Geometrie. Archive for History of Exact Sciences, 350(4), 329-344.

Torricelli, E. (1919-1944). Opera Geometrica. In Loria, G., and Vassura, G., editors. Opere di Evangelista Torricelli. Faenza, Italy: Stabilimento Tipografico Montanari. Italian translation by Luigi Belloni in Opere Scelte di Evangelista Torricelli, Torino, UTET, 1975.

Ulivi, E. (1977). Mode didattiche: il fusionismo. Archimede, 29, 211-216.

Valerio, L. (1606). Quadratura Parabolae per Simplex Falsum. Rome: Lepido Facii.

Van Cleve, J., \& Frederick, R. E., editors. (1991). The Philosophy of Right and Left: Incongruent Counterparts And The Nature Of Space. Dordrecht, The Netherlands: Kluwer.

Veblen, O. (1904). A system of axioms for geometry. Transactions of the American Mathematical Society, 50(3), 343-384.

Veblen, O., \& Young, J. W. (1916). Projective Geometry, Vol. 1. New York, NY: Blaisdell.

Veronese, G., \& Cazzaniga, P. (1897). Elementi di Geometria, ad uso dei licei e degli istituti tecnici (primo biennio). Verona, Italy: Fratelli Drucker.

Vitrac, B. (2000). Euclide, Les Élements, Volume IV, Livres XI-XIII: Géometrie des Solides, Traduction et Commentaire par Bernard Vitrac. Paris, France: PUF.

von Staudt, K. G. C. (1847). Geometrie der Lage. Nürnberg, Germany: F. Korn.

Webb, J. (1980). Mechanism, Mentalism, and Metamathematics. Dordrecht, The Netherlands: Reidel.

Webb, J. (2006). Hintikka on Aristotelian constructions, Kantian intuitions, and Peircian theorems. In Auxier, R. E., and Hahn, L. E., editors. The Philosophy of Jaakko Hintikka, Vol. XXX of The Library of Living Philosophers. Chicago, IL: Open Court.

Whitehead, A. N. (1906). The Axioms of Projective Geometry. London: Cambridge University Press.

Whiteside, D. T., editor. (1967). The Mathematical Papers of Isaac Newton. Vol. I: 16641666. London: Cambridge University Press.

Wiener, H. (1892). Über Grundlagen und Aufbau der Geometrie. Jahresbericht der Deutschen Mathematiker-Vereinigung, 1, 45-48.

\author{
DEPARTMENT OF PHILOSOPHY \\ 201 DICKENS HALL \\ KANSAS STATE UNIVERSITY \\ MANHATTAN, KS, 66503, USA \\ E-mail: andrew.arana@gmail.com \\ DEPARTMENT OF PHILOSOPHY \\ 314 MOSES HALL \#2390 \\ UNIVERSITY OF CALIFORNIA \\ BERKELEY, CA, 94720-2390, USA \\ E-mail:mancosu@socrates.berkeley.edu
}

